\title{
Crystallization of Covalent Organic Frameworks for Gas Storage Applications
}

\author{
Lijuan Zhu and Yue-Biao Zhang * \\ School of Physical Science and Technology, ShanghaiTech University, Shanghai 201210, China; \\ zhulj@shanghaitech.edu.cn \\ * Correspondence: zhangyb@shanghaitech.edu.cn; Tel.: +86-21-2068-5334
}

Received: 13 June 2017; Accepted: 4 July 2017; Published: 10 July 2017

\begin{abstract}
Covalent organic frameworks (COFs) have emerged as a new class of crystalline porous materials prepared by integrating organic molecular building blocks into predetermined network structures entirely through strong covalent bonds. The consequently encountered "crystallization problem" has been conquered by dynamic covalent chemistry in syntheses and reticular chemistry in materials design. In this contribution, we have reviewed the progress in the crystallization of COF materials and their hydrogen, methane and carbon dioxide gas storage properties for clean energy applications.
\end{abstract}

Keywords: covalent organic framework; crystallization; dynamic covalent chemistry; hydrogen storage; methane storage; $\mathrm{CO}_{2}$ capture

\section{Introduction}

In recent decades interest in organic materials with permanent nanometer scale pores has grown very quickly due to their specific properties and broad applications in gas storage, gas separation, drug delivery, energy conversion, catalysis, and optoelectronics. Scientists have designed and constructed a large number of porous materials, such as zeolites, metal-organic frameworks (MOFs), mesoporous silica, organosilica, microporous polymers, porous carbons, covalent organic frameworks (COFs) and organic molecular cages. These porous materials can be designed with variation of components, functionalities, pore metrics and skeletons. During the past decades, construction of persistent organic porous materials via covalent bonds is attracting increasing interest due to their unique stability and advanced applications. However, the wide pore-size-distribution and disordered extended structures of these amorphous networks restrict their further applications. Therefore, COFs as a class of covalent porous crystalline polymer in which the organic building blocks are covalently integrated to form extended ordered crystal structures, makes them different from the amorphous porous organic polymers [1-6]. The first synthetic challenge to make COFs, which was even thought impossible due to the difficulty of linking organic building blocks into crystalline extended structures entirely through strong covalent bonds, is the so-called "crystallization problem", the lack of solubility and reversibility for correcting errors during crystallization. Such difficulties were first conquered by the judicious choice of reversible condensation reactions where water is the byproduct, whose retention can be further used to control the direction of reactions for good reversibility. Meanwhile, the bond energy is also a key parameter for the ease of bond breaking and reformation during crystallization. It is worth mentioning that it is easy to used weaker covalent bonds to achieve higher crystalline products, but the framework's robustness necessary to achieve practical functions, such as permanent porosity and structure integrity in applications, would then be compromised. Obviously, there is a trade-off between crystallinity and robustness in making COF in the current state of the art. We do believe, however, that this situation will be resolved in the near future by knowing how to control the reversibility and the precision of molecular design. 


\section{Dynamic Covalent Chemistry of COFs}

In order to achieve higher reversibility for efficient error correction during crystallization, dynamic covalent chemistry (DCC) has been well applied in the construction of COFs. To stitch discrete molecular building blocks into periodic networks, DCC enables strong covalent bond formation accompanied by the assembly of molecular building blocks into predetermined spatial arrangements $[7,8]$. Considering the possible spatial arrangements of the moiety formed in the reaction, there are multiple products in the equilibrium with various degrees of crystallinity. Such reversible assembly of molecular components can proceed along kinetic or thermodynamic pathways (Scheme 1). Due to the lower barrier of activation $\left(\Delta G^{\ddagger}\right)$, kinetic intermediates dominate initially due to a fast rate of formation the kinetic products, which can be trapped during quenching of the reaction and form amorphous products with the gain of entropy from their randomness of arrangement, meanwhile the thermodynamic products have the lowest overall Gibb's free energy $\left(\Delta G^{\circ}\right)$, so that given the opportunity the reaction will re-equilibrate towards the global minimum to form the most stable and probably crystalline products. It is also possible that the kinetic products can also be transformed into thermodynamic products by using certain thermo-treatments for recrystallization. It is common in COF chemistry that amorphous products are initially obtained until the synthetic conditions are optimized by varying the crystallization times, reaction temperatures, reaction solvents, and catalysts (such as acids, bases, and metal salts acting as Lewis acids). The discovery process may change the activation energy of thermodynamic products and their reaction rates to accelerate the crystallization of COFs. On the other hand, rational design of building blocks may also increase the correctness of the assembly of molecular components, thus enhance the crystallinity of the obtained products. It is generally believed that $2 \mathrm{D}$ COFs are easier to form having less probability of resulting in networks than the 3D ones with more structural diversity.

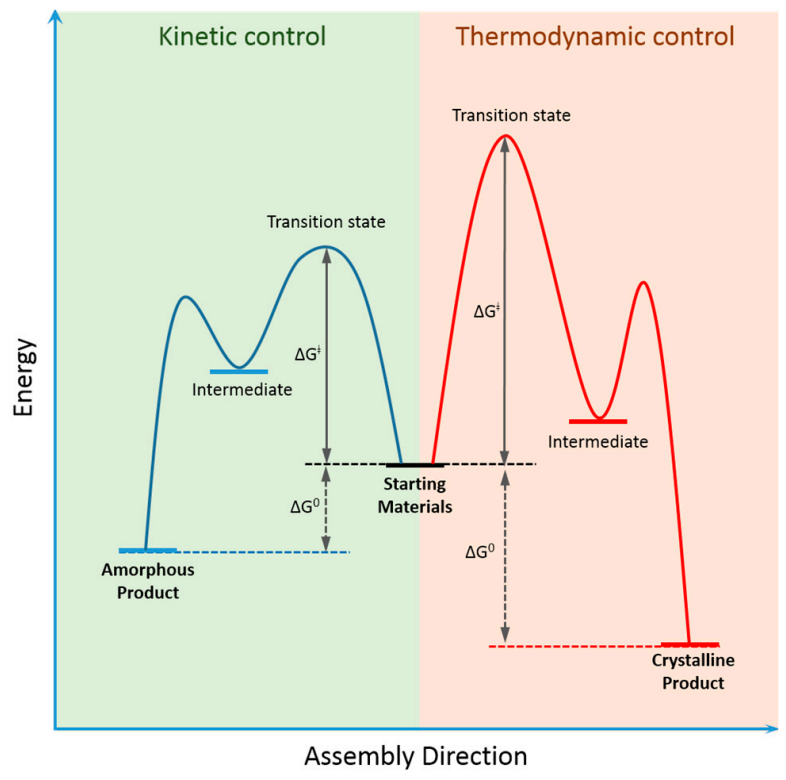

Scheme 1. Thermodynamic control in the dynamic covalent chemistry of COFs.

From the chemistry viewpoint, the difficulty of constructing COF materials can be classified by the difference of bond energy of the linkages between the molecular building blocks (Figure 1). It is easier for weak covalent bonds to break and re-form to correct assembly errors, such as the reversible covalent bond have enabled to make products with large single crystals [9]. However, the lack of robustness prevents their permanent porosity for further applications. For stronger covalent bonds, their reversibility during the reactions are highly dependent on the reaction conditions, which can perform both crystallinity and robustness after isolation from the system. 
B-O linkages

Borobate Ester

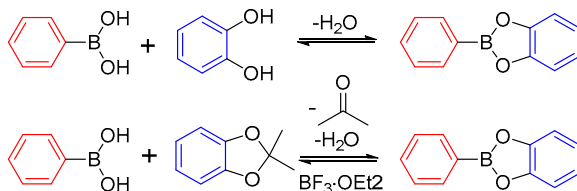

Borosilicate

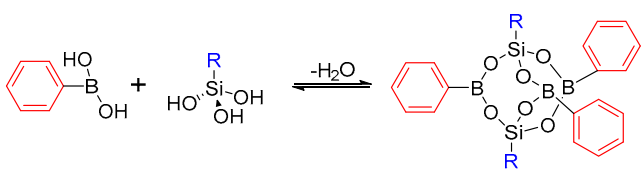

\section{C-N linkages}

\section{Imine}<smiles>Oc1ccc(/C=N/c2ccccc2)cc1</smiles>

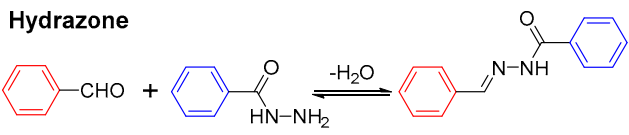

Azine

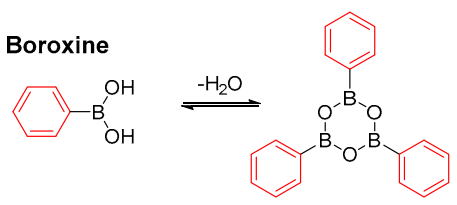

Spiroborate

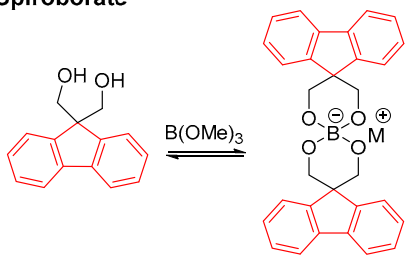

Squaraine
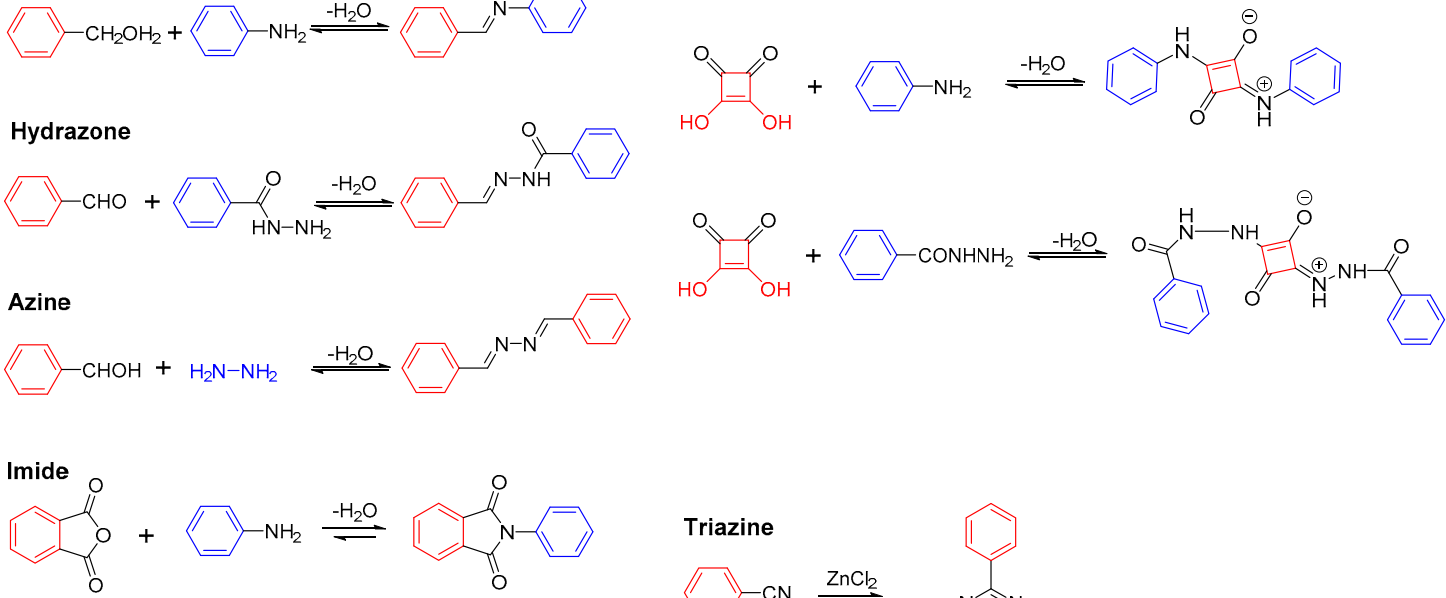

Phenazine
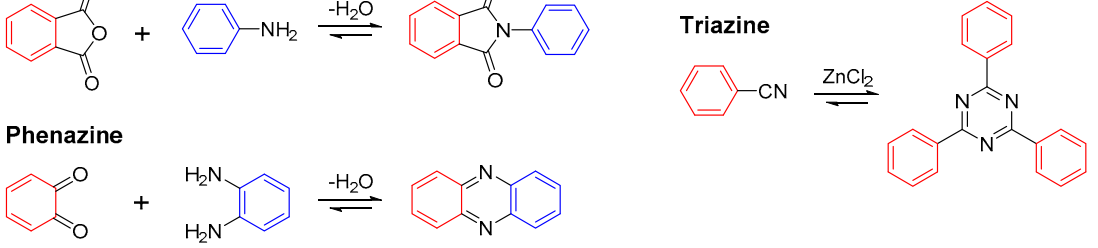

\section{Secondary Amine}
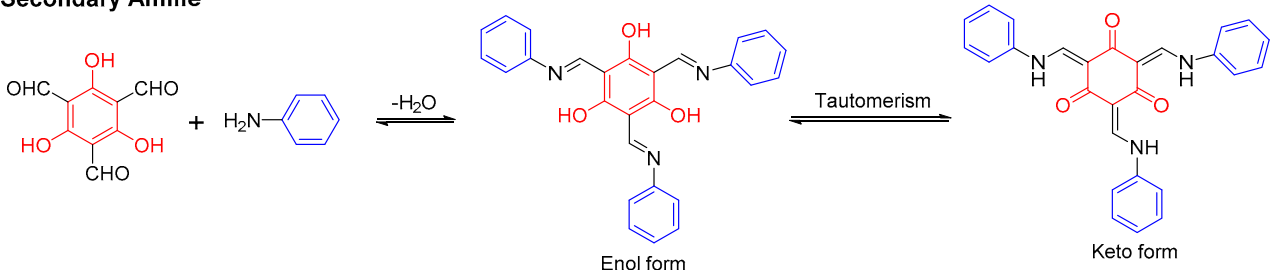

Amide

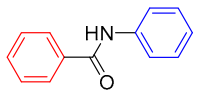

Figure 1. Several reversible reactions used for construction of COF materials. Dynamic reversible reactions are essential for the formation of crystal covalent organic frameworks (COFs). These reversible reactions for the formation of COFs are boron-containing linkages (for example, boroxine, boronate ester, borazine and spirobrate), nitrogen-containing linkages (for example, imine, hydrazone, azine, squaraine, phenazine, triazine, imide and azodioxy), and a double-stage linkage containing the boronate ester linkage and the imine linkage in one COF via a one-pot reaction.

\subsection{B-O Linkages}

Featuring light-weight compositions and high crystallinity, boron-based COFs have been intensively studied including understanding their crystallization mechanism, to the designing of new 
topologies, achieving high surface area, and the utilization in gas storage applications. The successful construction of such crystalline materials benefited from the decent reversibility of the B-O linkages, including boroxine $\left(-\mathrm{B}_{3} \mathrm{O}_{3}\right)$, dioxaborole $\left(-\mathrm{BO}_{2} \mathrm{C}_{2}\right)$, and spiroborate $\left(-\mathrm{O}_{4} \mathrm{~B}^{-}\right)$.

\subsubsection{Boroxine-Linked COFs}

The emergence of COF chemistry was triggered by Yaghi and co-workers' seminal work on the self-condensation of aromatic polyboronic acids (Figure 2) [10]. The successful crystallization of these materials was attributed to the implementation of a closed reaction system to sustain the availability of water for maintaining reversible conditions conducive to crystal growth. The solvents used and their mixture are a handle to control the diffusion of building blocks into the crystallization mother liquor. The crystallization was intentionally processed slowly and terminated after sufficient time had elapsed.
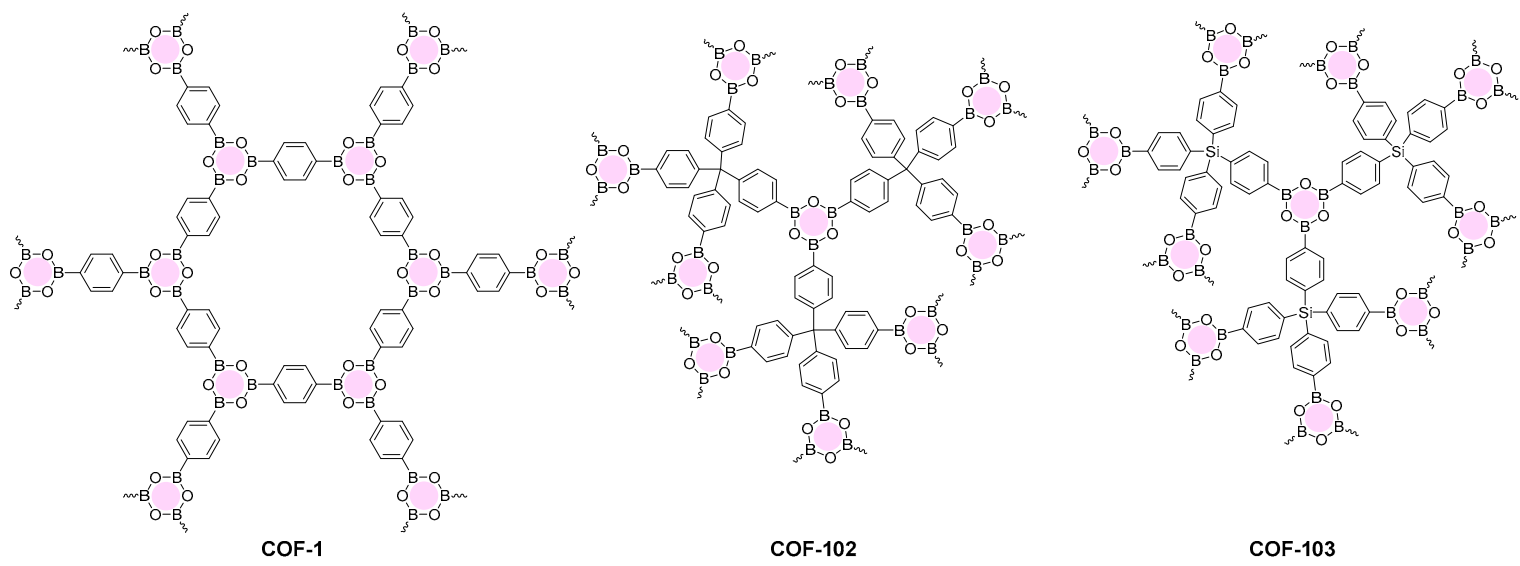

Figure 2. Chemical structures of classic boron-containing COFs (COF-1, COF-102, COF-103) with boroxine connections.

The choice of solvent and temperature to maximize the error correction associated with the boroxine ring-forming reactions can optimize the crystallinity of the boroxine-based COFs. COF-1 with two-dimensional (2D) architectures, was prepared by the self-condensation of 1,4-phenylenediboronic acid (BDBA) under solvothermal conditions, to produce an extended staggered layered structure with hexagonal pores of $15 \AA$ diameter and a BET surface area of $711 \mathrm{~m}^{2} \cdot \mathrm{g}^{-1}$. Yaghi and co-workers also synthesized three-dimensional (3D) COFs, COF-102 and COF-103 by self-condensation of tetrahedral (3D- $T_{\mathrm{d}}$ ) nodes, tetra(4-dihydroxyborylphenyl)methane (TBPM) and its silane analog (TBPS), respectively (Figure 2) [11]. These two COFs are the crystal porous organic frameworks with high surface areas (3472 $\mathrm{m}^{2} \mathrm{~g}^{-1}$ for COF-102 and $4210 \mathrm{~m}^{2} \mathrm{~g}^{-1}$ for COF-103) and pore size distributions of $11.5 \AA$ (COF-102) and $12.5 \AA$ (COF-103).

Microwave heating can accelerate the reaction times and obtain much cleaner COFs with high yields. Cooper and co-workers demonstrated the synthesis and purification of COF-5 and COF-102 by microwave heating. The microwave synthetic method reported by Cooper and co-workers was 200 times faster than solvothermal methods reported by Yaghi et al. without changing the physical properties of COFs [12].

\subsubsection{Dioxaborole-Linked COFs}

The cross-condensation of polybornic acids with catechols opened the way to stich different building blocks into one framework (Figure 3). The popular utilization of 2,3,6,7,10,11-hexahydroxytriphenylene (HHTP) as a triangular building block with ditopic, tirtopic and tetratopic boronic acids leads to 2D and 3D networks (Figure 3) $[10,11,13]$. With a sealed reaction system, COF-5, $-6,-8$ and -10 were 
successfully prepared in the cocktail solvent system by mixing mesistylene and 1,4-dioxane for 3-5 days at $85-100{ }^{\circ} \mathrm{C}[10,13]$. A similar COF with the same HHTP as co-linker can be also prepared by stirring under an inert atmosphere [14].

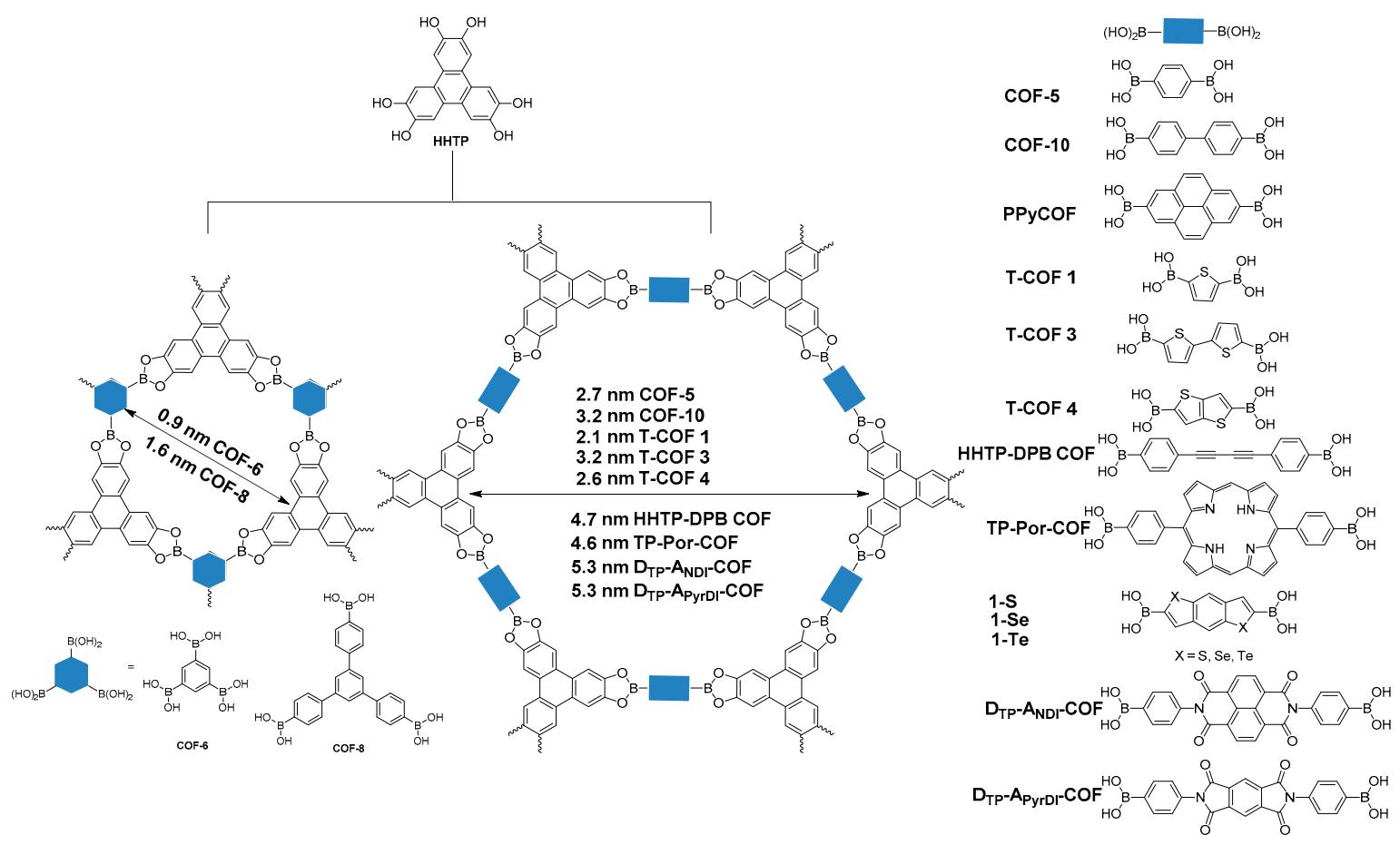

Figure 3. Construction of dioxaborole-linked COFs via the cross-condensation of triangular HHTP with ditopic or tritopic boronic acids to form 2D honeycomb layer structures.

By using the tetrahydroxybenzene and its derivatives, 2D COFs can be prepared by stitching tritopic boronic acids together. The reactions were conducted in the mixture of tetrahydrofuran (THF) and methanol $(\mathrm{MeOH})$ by stirring under an inert atmosphere $[15,16]$.

Boronate ester linked COFs have high crystallinity, but low hydrolytic stability and chemical stability because of the reversibility of the reactions, which leads to decomposition upon exposure to water or acid. This disadvantage greatly limits their applications. Alkylation in the pores of COFs improves the stability by decreasing the hydrolysis rate of the connected linkage. Lavigne and co-workers were the first to incorporate alkyl groups into the channel walls during the synthesis of the boronate ester linked COFs to increase the stability of COFs, as well as to fine tune the pore sizes [16]. The presence of alkyl chains in the channels control the pore size from $1.8 \mathrm{~nm}$ to $1.1 \mathrm{~nm}$ (Figure 4), which were observed for the series of COF-18 $\AA$ (no alkyl group), COF-16 $\AA$ (methyl), COF-14 $\mathrm{A}$ (ethyl), and COF-11 $\AA$ (propyl). Meanwhile, the surface area decreased from 1263 to $105 \mathrm{~m}^{2} \cdot \mathrm{g}^{-1}$ and the pore volume decreased from 0.69 to $0.052 \mathrm{~cm}^{3} \cdot \mathrm{g}^{-1}$, as the chain length increased. Interestingly, modification of the pore interior with increasingly larger alkyl groups causes a decline in nitrogen uptake but an increase in the molar amount of hydrogen adsorbed. Once submersion in aqueous media, the porosity of alkylated COFs decreased by about $25 \%$, while the non-alkylated COFs were almost completely hydrolyzed, losing virtually all porosity. In addition, the degree of crystallinity decreased by about $40 \%$ for alkylated COFs and $95 \%$ for non-alkylated COFs. By using microwave synthesis, Bein et al. extended the framework into materials having large $4 \mathrm{~nm}$ openings (Figure 4), featuring one of the largest pores in crystalline materials at this time [17]. 

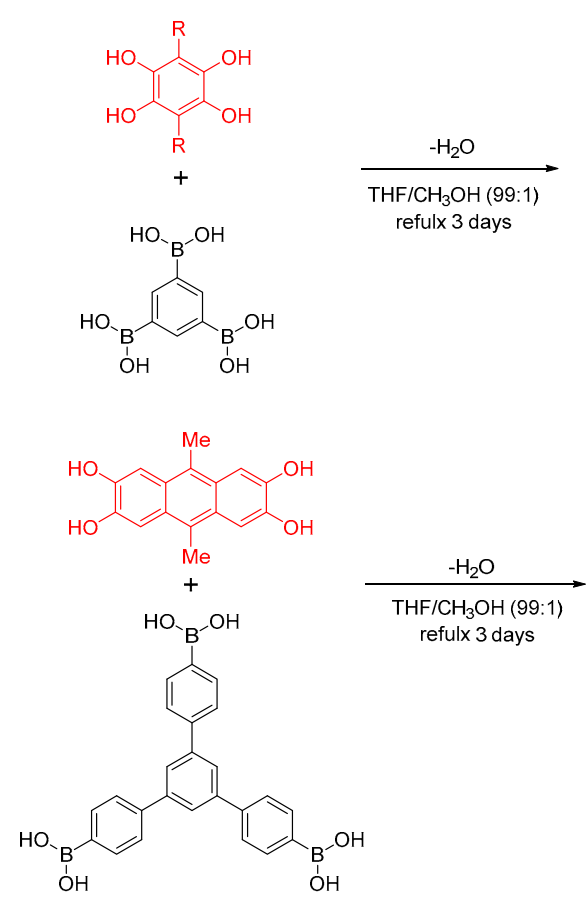
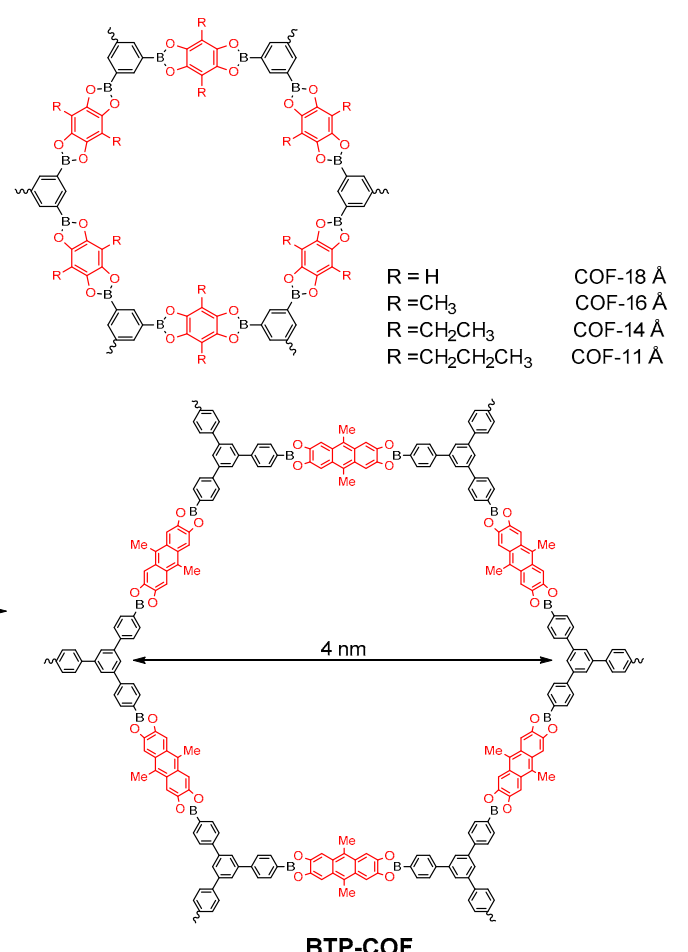

Figure 4. Construction of dioxaborole-linked COFs via the cross-condensation of ditopic catechol-based linkers with tritopic boronic acids to form the $2 \mathrm{D}$ honeycomb layer structures. The pore sizes can be modulated by the decorated functionalities of the ditopic linkers, and expanded by elongating both the boronic acid and catechol linkers.

By using octahydroxyphthalocyanine and its metalated version, the Dichtel group was able to expand the pore size of the ZnPc lattice from 2.7 to $4.4 \mathrm{~nm}$ (Figure 5) [18]. The Dichtel group also reported the selective and patterned growth of a $2 \mathrm{D}$ phthalocyanine COF on single-layer graphene. This provides a promising pathway to direct the growth of COF films [19].

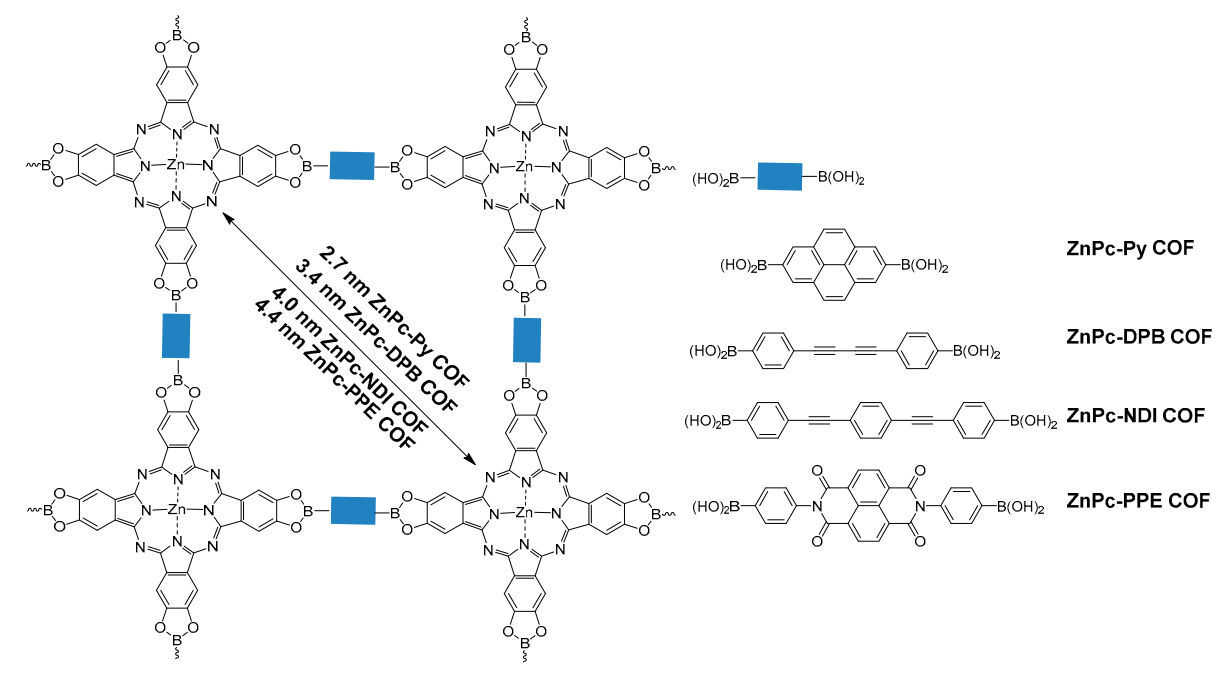

Figure 5. Construction of dioxaborole-linked COFs via the cross-condensation of ditopic catechol-based linkers with tritopic boronic acids to form $2 \mathrm{D}$ honeycomb layer structures. The pore sizes can be modulated by the decorated functionalities of the ditopic linkers, and expanded by elongating both the boronic acid and catechol linkers. 
To avoid the oxidation and insolubility of polycatechols, protected catechols were proposed as starting materials for making COFs which can be deprotected in-situ under catalysis by a Lewis acid such as $\mathrm{BF}_{3} \cdot \mathrm{OEt}_{2}$ [20]. With this strategy, triangular HHTP and square phthalocyanine as well as its metalated derivatives have been successfully incorporated into boronate ester-linked COFs (Figure 6). Mechanistic studies show that before adding the Lewis acid, the boronic acids self-condensate reversibly to form boroxine-linked products and $\mathrm{H}_{2} \mathrm{O}$. The addition $\mathrm{of}^{\mathrm{B}} \mathrm{BF}_{3} \cdot \mathrm{OEt}_{2}$ catalyzes acetonide hydrolysis of protected catechols, and the resulting catechol will rapidly condense with the boronic acids to form the boronate-linked COFs [21]. Once the free boronic acids are consumed, the formed products will hydrolyze and release more boronic acids, which will produce more boronate-linked COFs.
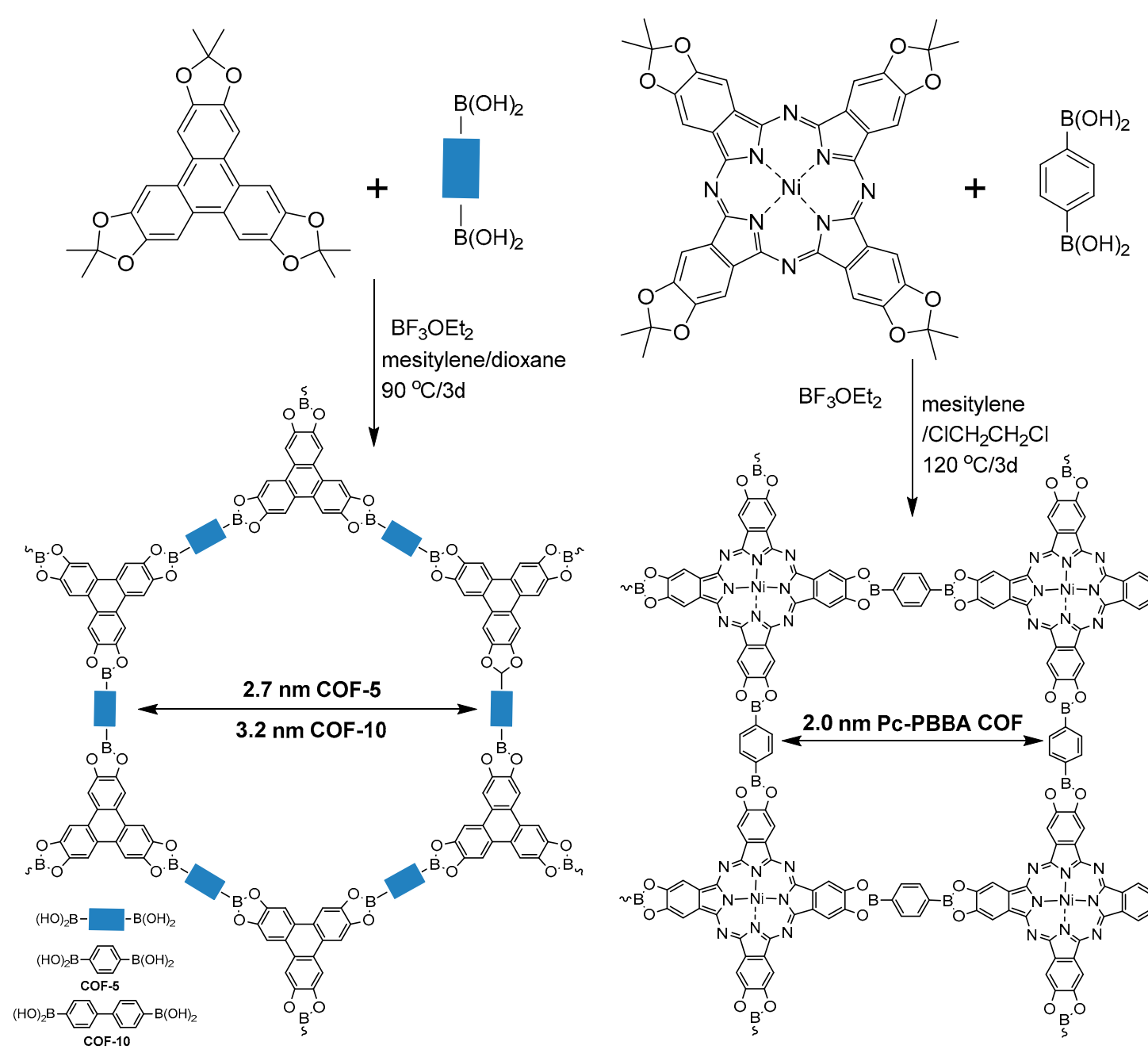

Figure 6. Synthesis of dioxaborole-linked COFs starting from protected catechol-based linkers with boronic acids for improved solubility and anti-oxidation in comparison with pristine catechol linkers.

To accelerate the synthesis of highly crystalline COF material, a two-step microwave synthesis has been proposed for protected boronic acids as starting materials with the addition of HHTP (Figure 7). The resulting product proved to be highly crystalline and possess large pore openings. Control experiments didn't yield COF via the one-pot reaction. This work also provided the first high-resolution TEM image of a COF showing the pores clearly [22]. 

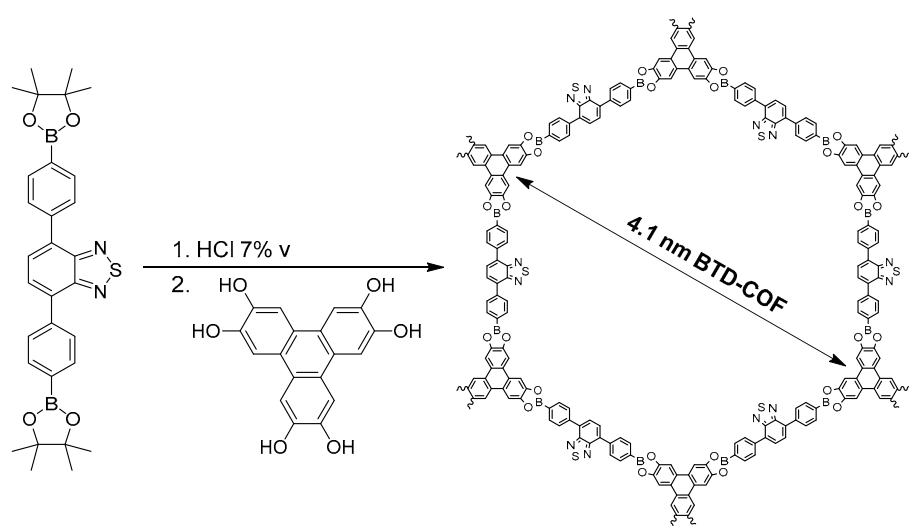

Figure 7. Synthesis of the dioxaborole-linked COF by in-situ deprotection of boronic pinacol ester followed with the addition of HHTP via microwave heating method.

A modulation approach has been developed to control the structure and crystallinity of COF materials, introducing a modulator in the synthesis that can compete with one of the building blocks during the solvothermal COF growth to form highly crystalline frameworks with large domain size and very high porosity. Bein and co-workers used monoboronic acids as modulators in the solvothermal synthesis of the archetypical COF-5 to optimize the crystallinity, domain size, and porosity of 2D COFs (Figure 8). Moreover, the addition of the monoboronic acids also provides a potential construction of functional crystalline COF materials [23].

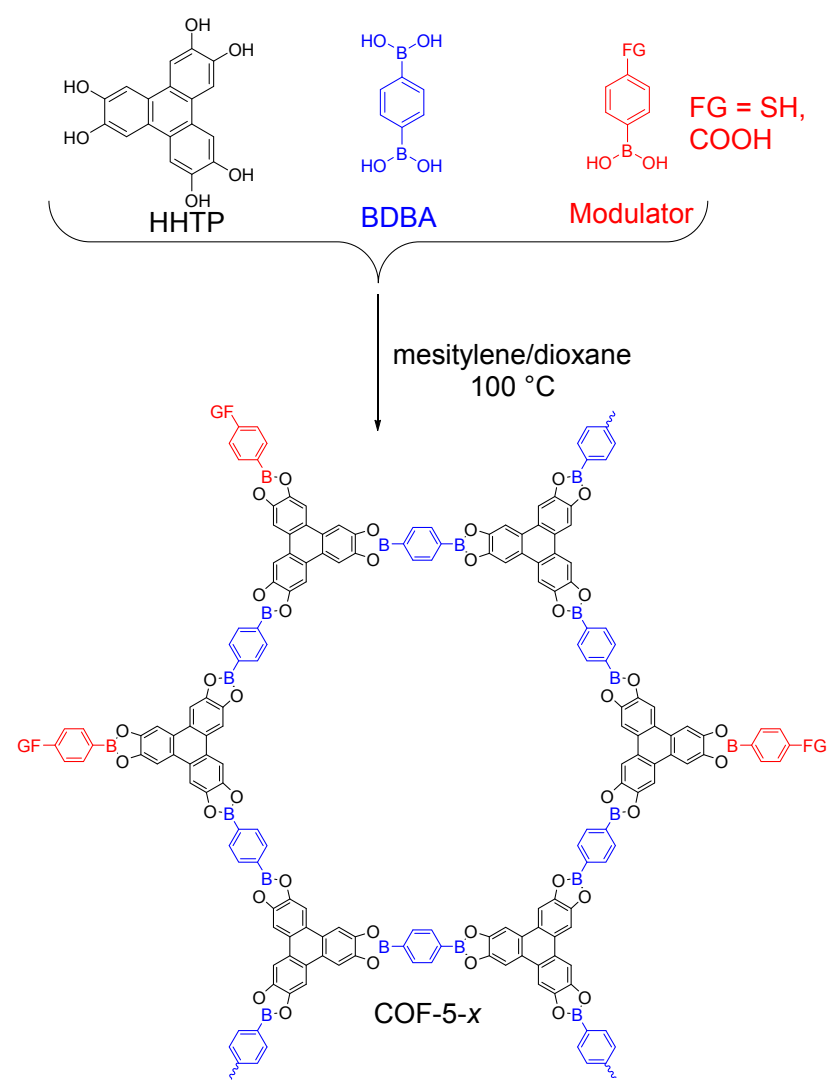

Figure 8. The modulation approach to synthesize COF-5- $X$ via the cross-condensation of HHTP and 1,4-diboronic acid combining with terminal boronic acids with various functional groups to achieve higher crystallinity and external functionalities. 


\subsubsection{Spiroborate-Linked COFs}

Researchers have shown great interest in exploring new methods to enhance the chemical stability of boron-containing COFs. Recently, the condensation of diols with trialkyl borate in the presence of basic catalysts has been explored for the synthesis of spiroborate-linked ionic COFs in which the negatively charged borons are located on the edges with different cations as counter ions. Zhang and co-workers [24] constructed a novel type of spiroborate-linked ionic covalent organic framework (ICOF, Figure 9), which contains $\mathrm{sp}^{3}$ hybridized boron anionic centers and tunable countercations (lithium or dimethylammonium). The synthesized ICOFs (ICOF-1 and ICOF-2) show good thermal stabilities and excellent resistance to hydrolysis, remaining nearly intact when immersed in water or basic solution for up to two days. The SEM images support the single-crystalline morphology of ICOF-1 and ICOF-2. The PXRD results also show multiple sharp peaks, which indicate the orderliness structures in the framework. However, the crystal structures of ICOFs have not been characterized. These ICOFs also have high BET surface areas up to $1259 \mathrm{~m}^{2} \cdot \mathrm{g}^{-1}$ and adsorb a significant amount of $\mathrm{H}_{2}$ (up to $3.11 \mathrm{wt} \%, 77 \mathrm{~K}, 1 \mathrm{bar}$ ) and $\mathrm{CH}_{4}$ (up to $4.62 \mathrm{wt} \%, 273 \mathrm{~K}, 1$ bar). The existence of permanently immobilized ion centers in ICOFs enables the transportation of lithium ions with room-temperature lithium-ion conductivity of $3.05 \times 10^{-5} \mathrm{~S} \cdot \mathrm{Lcm}^{-1}$ and an average $\mathrm{Li}^{+}$ transference number value of $0.80 \pm 0.02$.

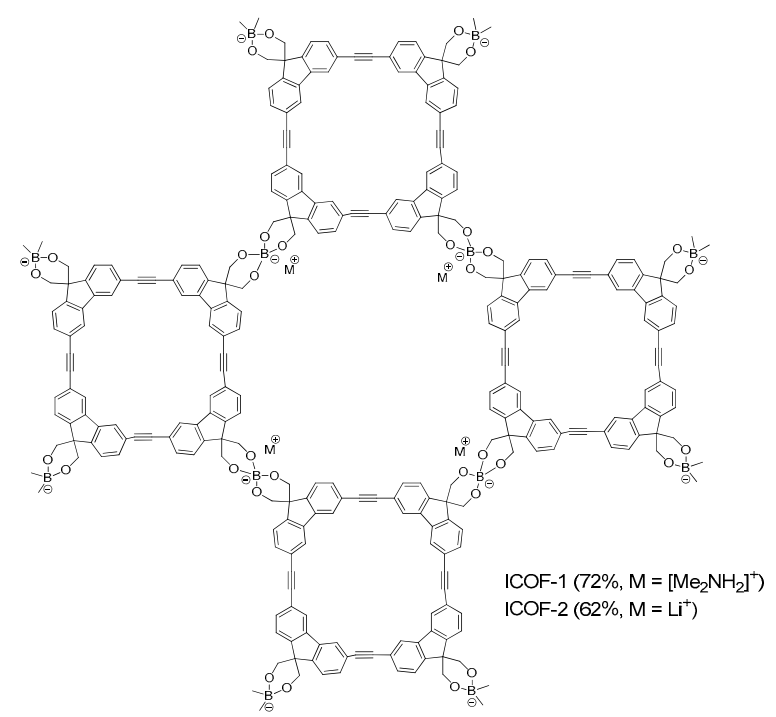

Figure 9. Construction of spiroborate-linked COFs featuring anionic skeletons with various counter ions for high ionic conductivity.

\subsection{C-N Linkages}

\subsubsection{Imine-Linked COFs}

To avoid the structural fragility of boron-containing COFs, imine-based COFs were discovered by a reversible condensation of polytopic anilines with polybenzaldehydes or polyketone with elimination of water by a catalyst of aqueous acetic acid (Figure 10). Generally, the crystallinity of the imine-based COFs is lower compared with that of boronate ester-linked COFs, whereas the chemical stability in the presence of water, acids and bases is significantly enhanced.

The first imine-linked COF was developed by Yaghi and co-workers in 2009 [25]. They constructed an imine-based COF (COF-300), which possesses a 3D five-fold interpenetrating diamond-like structure. COF-300 has a BET surface area of $1360 \mathrm{~m}^{2} \cdot \mathrm{g}^{-1}$ and a pore size of $7.8 \AA$, which provides better hydrolytic stability compared to the boron-containing COFs. Moreover, the broad range 
of multifunctional amines and aldehydes provides a large number of structural possibilities for imine-based COFs.

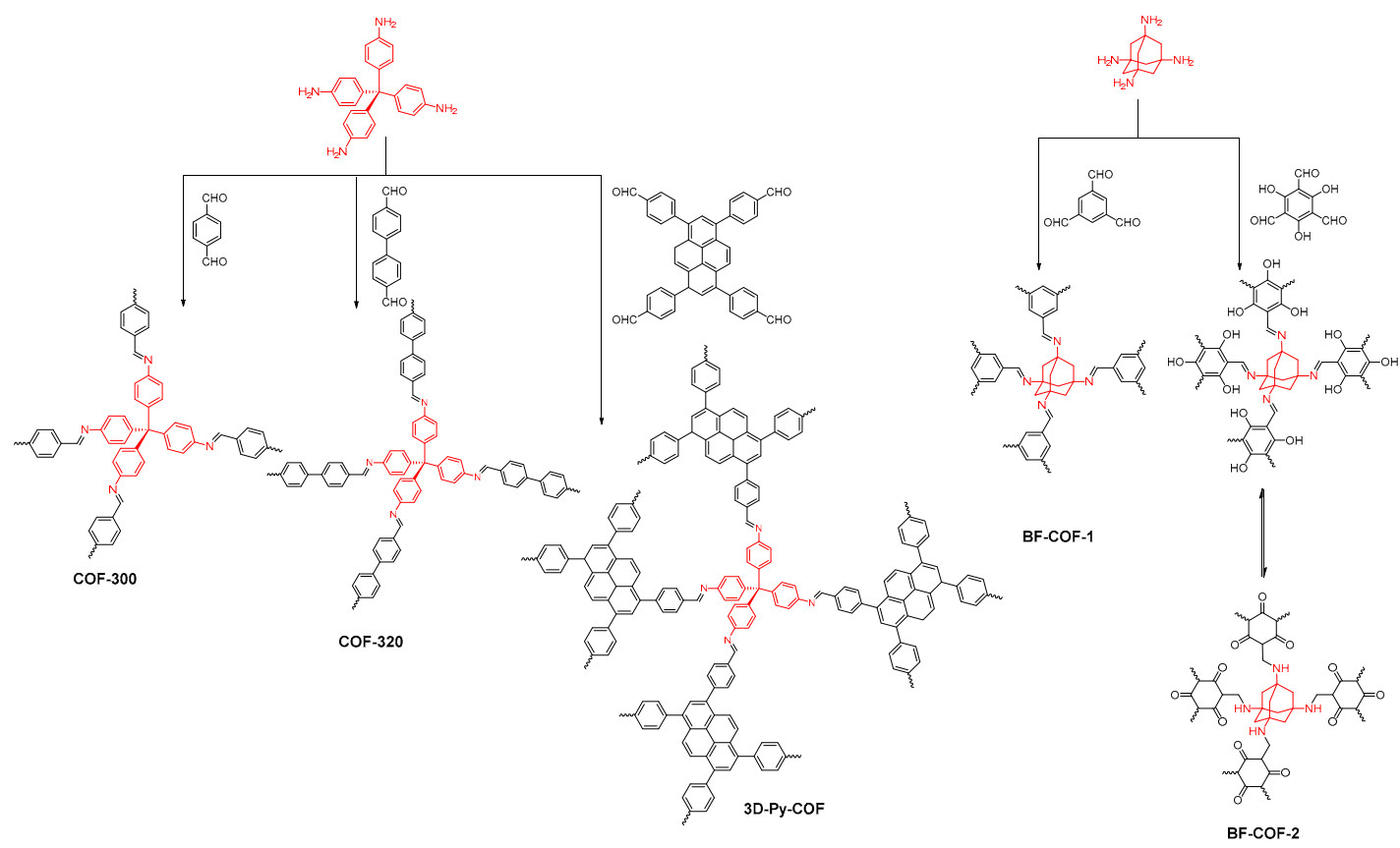

Figure 10. Construction of imine-linked COFs by co-condensation of tetratopic anilines (tetra-(4-anilyl)methane, and 1,3,5,7-tetraaminoadamantane) with various ditopic, tetraaldehydes and triangular aldehydes to form 3D networks.

Imine formed COF-320 was prepared through solvothermal condensation of tetra-(4-anilyl)-methane and 4,4'-biphenyldialdehyde in 1,4-dioxane at $120^{\circ} \mathrm{C}$. A 9-fold interlaced diamond network was formed and the final crystalline material exhibits high porosity with a Langmuir surface area of $2400 \mathrm{~m}^{2} \cdot \mathrm{g}^{-1}$. The crystal structure of the COF-320 solid was elucidated by single-crystal 3D electron diffraction [26]. Recently, a pyridyl functionalized version of COF-320 was prepared, namely LZU-301, in which the reversible dynamic response upon guest accommodation and release was uncovered. Thus, the design principle of 3D dynamic COFs was claimed [27].

Wang and co-workers synthesized a novel 3D pyrene-based COF (3D-Py-COF) by condensation of tetra(p-aminophenyl)methane and 1,3,6,8-tetrakis(4-formylphenyl)-pyrene. This 3D-Py-COF has a two-fold interpenetrated pts topology, which was first reported in this work. Due to the existence of pyrene units in the 3D frameworks, 3D-Py-COF exhibits fluorescent properties and can be used in explosives detection [28].

Yan and co-workers designed and synthesized two new 3D base-functionalized COFs with micropores (BF-COF-1 and BF-COF-2), by the reaction of a tetrahedral alkyl amine, 1,3,5,7-tetraaminoadamantane (TAA), and 1,3,5-triformylbenzene (TFB) or triformylphloroglucinol (TFP) [29]. These BF-COFs were used for the Knoevenagel condensation reaction with high conversion (BF-COF-1: 96\% and BF-COF-2: 98\%), highly efficient size selectivity, and good recyclability. The structural rigidity of the admantane building unit is the key for forming targeted products. In contrast, the combination of tetra( $p$-aminophenyl) with TFB could not produce crystalline product.

To enhance both the chemical stability and crystallinity in $2 \mathrm{D}$ porphyrin COFs, Banerjee and co-workers proposed a new strategy to protect the COF interior by introducing -OH to the Schiff base $[-\mathrm{C}=\mathrm{N}]$ centers in $\mathrm{COF}$ and creating an intramolecular $[\mathrm{O}-\mathrm{H} \cdots \mathrm{N}=\mathrm{C}]$ hydrogen bond (Figure 11) [30]. This hydrogen-bonding interaction enhances the stability of imine bonds in the presence of water and acid $(3 \mathrm{~N} \mathrm{HCl})$. Otherwise, this hydrogen bond in DhaTph also could enhance the crystallinity and porosity, compared to the methoxy-substituted COF DmaTph in which this intramolecular hydrogen 
bond does not exist. This work demonstrates that the introduction of intramolecular hydrogen bonds can not only improve the chemical stability, but also the crystallinity and porosity of 2D COFs, which would broaden the potential applications of COF materials.

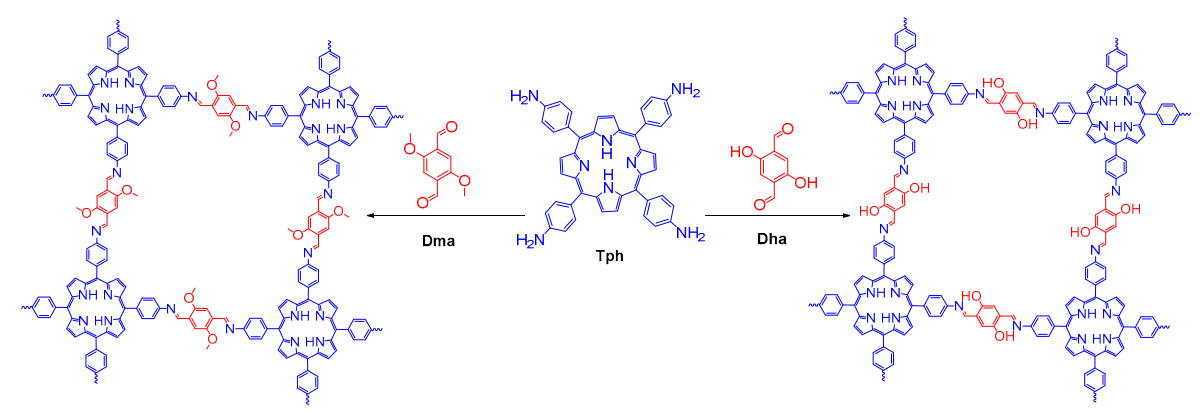

Figure 11. Syntheses of DmaTph and DhaTph by the condensation of square planar Tph building unit (blue) and linear Dma/Dha building unit (red). The introducing of $-\mathrm{OH}$ to the Schiff base [-C=N] centers in COFs can create an intramolecular $[\mathrm{O}-\mathrm{H} \cdots \mathrm{N}=\mathrm{C}]$ hydrogen bond to enhance both the chemical stability and crystallinity of COFs.

The formation of crystalline structures via relatively weak interactions between planar layers has been well defined and designed in crystal engineering. Jiang and co-workers synthesized a series of 2D COFs locked with intra-layer hydrogen-bonding (H-bonding) interactions (Figure 12) [31]. The H-bonding interaction sites were located on the edge units of the imine-linked tetragonal porphyrin COFs, and the contents of the H-bonding sites in the COFs were synthetically regulated by using a three-component condensation system. The intra-layer H-bonding interactions suppress the torsion of the edge units and lock the tetragonal sheets in a planar conformation. This planarization enhances the interlayer interactions and triggers extended $\pi$-cloud delocalization over the $2 \mathrm{D}$ sheets. Upon AA stacking, the resulting COFs with layered 2D sheets amplify these effects and strongly affect the physical properties of the material, including improving their crystallinity, enhancing their porosity, increasing their light-harvesting capability, reducing their band gap, and enhancing their photocatalytic activity toward the generation of singlet oxygen.

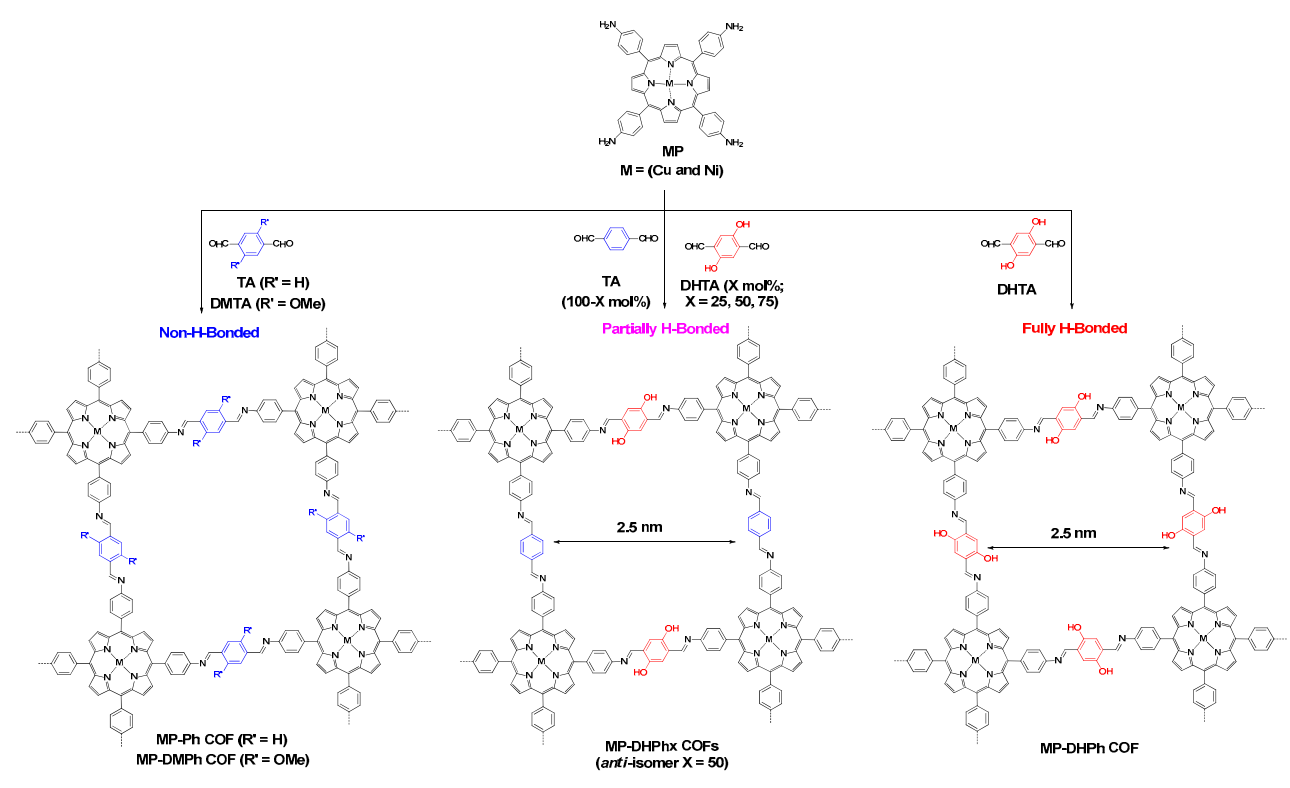

Figure 12. Schematic of the synthesis of $2 \mathrm{D}$ porphyrin COFs with designable content of hydrogen-bonding structures. 
In addition to $\mathrm{H}$-bonding interactions, self-complementary $\pi$-electronic forces also provide new opportunities for enhancing the crystallinity of COFs. Jiang and co-workers reported a synthetic method to control the crystallinity and porosity of COFs by managing interlayer interactions based on self-complementary $\pi$-electronic forces (Figure 13).
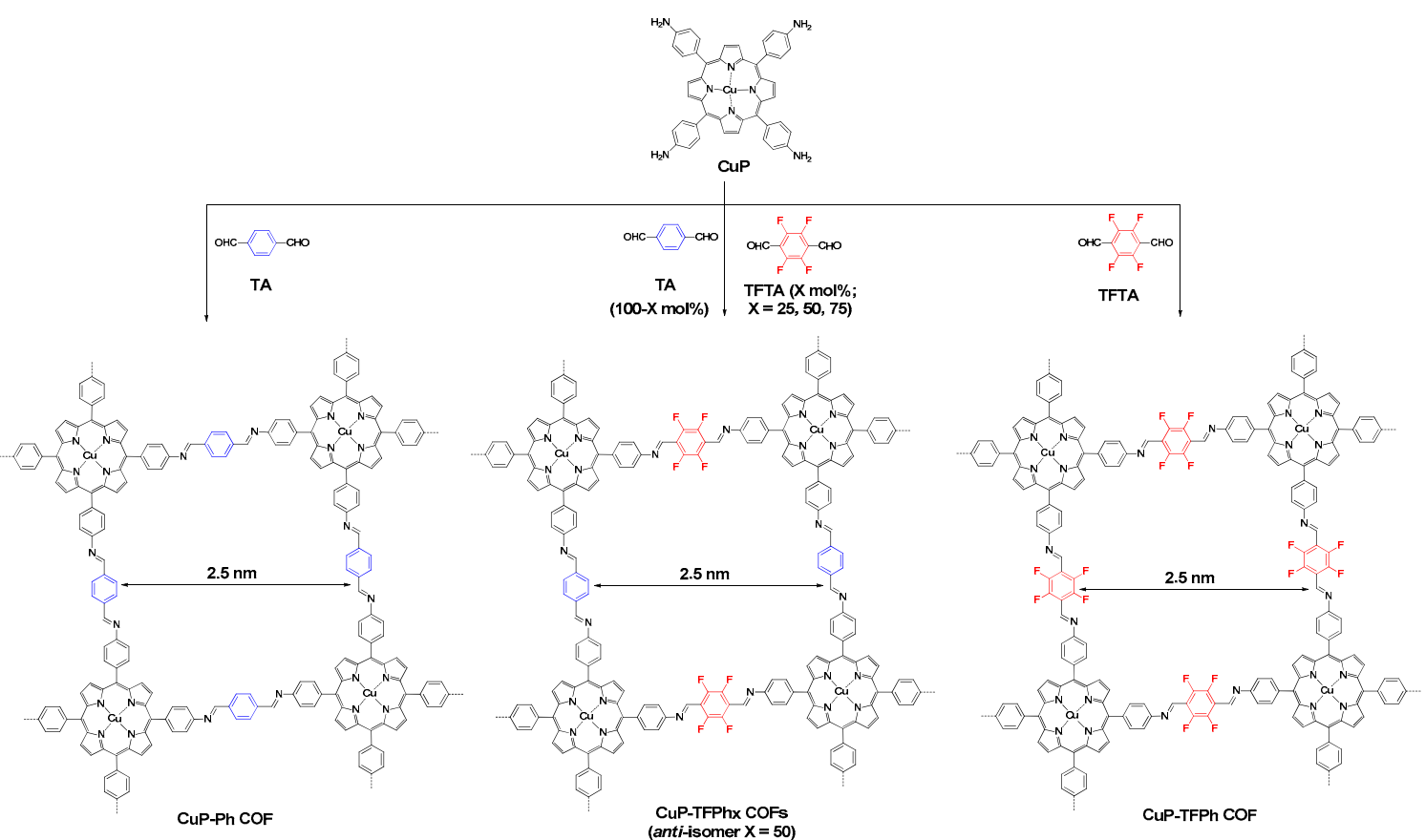

Figure 13. Schematic of the synthesis of $2 \mathrm{D}$ COFs integrated with self-complementary $\pi$-electronic force $\left(\mathrm{CuP}-\mathrm{TFPh}_{\mathrm{X}}, \mathrm{X}=25,50\right.$, and $\left.75 \mathrm{~mol} \%\right)$ and the $\mathrm{CuP}-\mathrm{Ph}$ and $\mathrm{CuP}-\mathrm{TFPh}$ controls.

They controlled the crystal structure of CuP-Ph COF by introducing 2,3,5,6-tetrafluoroterephthalaldehyde into the $\mathrm{COF}$ structure. Fluoro-substituted aromatic units in different ratios were integrated into the edge units, which will induce the self-complementary $\pi$-electronic interactions in the COFs. This interaction increases the crystallinity and the porosity of COFs by maximizing the total crystal stacking energy and minimizing the unit cell size. This work provides a new pathway to improve the crystals of COFs by controlling the interlayer interactions [32].

The stability and crystallinity of $2 \mathrm{D}$ COFs are highly related to the factors of the interlayer interactions between the $2 \mathrm{D}$ layers. In imine-based $\mathrm{COFs}$, the $\mathrm{C}=\mathrm{N}$ bond is polarized to yield partially positively charged carbon and negatively charged nitrogen, which will cause electrostatic repulsion between the layers and could destabilize the layered structure staking. Jiang and co-workers introduced methoxy groups (-OMe) into the pore walls to reinforce the interlayer interactions (Figure 14). The oxygen lone pairs on the methoxy-substituted $C_{2}$-phenyl edges soften the polarization through resonance and increase the stability and high crystallinity of the COF. Compared to the TPB-TP-COF and TPB-DHTP-COF, the methoxy-functionalized TPB-DMTP-COF has much higher crystal stacking energy, showing high crystallinity and high BET surface area. Through the introduction of chiral centers, (S)-Py moieties, onto the walls of the open channels, they obtained chiral COFs which can be used for heterogeneous asymmetric organocatalysis of asymmetric Michael reactions. The Jiang group also investigated the proton conduction properties of this highly crystalline and stable COF and explored the potential applications in many areas, including fuel cells, electrochemical sensors, electrochemical reactors and electrochromic devices [33].From Figure 15, we can observe that TPB-DMTP-COF also exhibits much higher BET and Langmuir surface area compared to the COFs without enhanced interlayer interactions and hydrogen bond enhanced COFs. As shown in Figure 15, the BET surface area decreases with the decrement of the methoxy group ratio, which demonstrates 
that the introduced methoxy groups greatly improve the crystallinity of the COFs. This kind of $\mathrm{COF}$ also shows enhanced chemical stability. After immersing in 12-M HCl, boiling water and 14-M $\mathrm{NaOH}$ for 1 week, the surface area of TPB-DMTP-COFs still retains the original values. However, the non-methoxy group-based COFs (TPB-TP-COF and TPB-DHTP-COF) show very week acid, base or boing water tolerance.

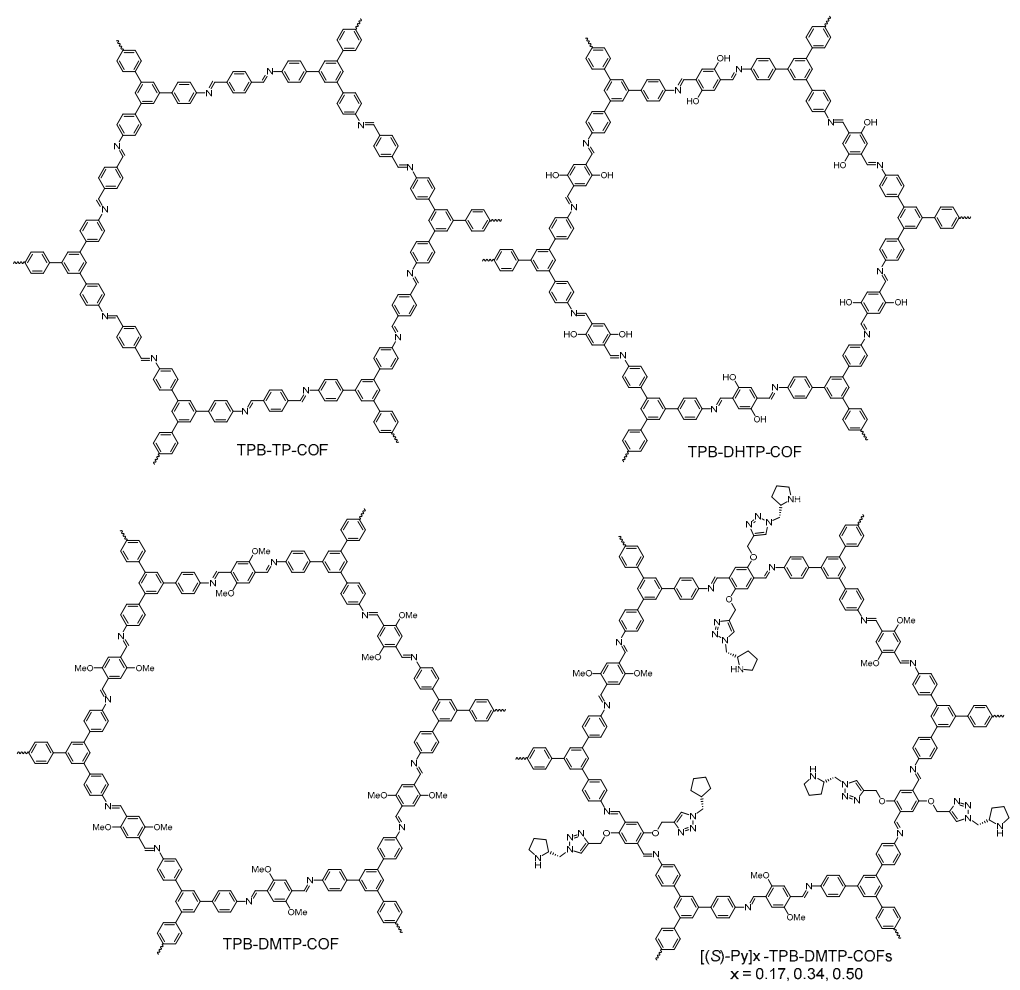

Figure 14. Synthesis and structure of stable crystalline porous COFs through softened interlayer charge repulsion. (a) TPB-TP COF control; (b) TPB-DHTP COF control; (c) stable crystalline porous COF of TPB-DMTP COF; (d) functionalization of TPB-DMTP COF ([(S)-Py $] x-$ TPB-DMTP-COFs, $x=0.17$, $0.34,0.50)$.
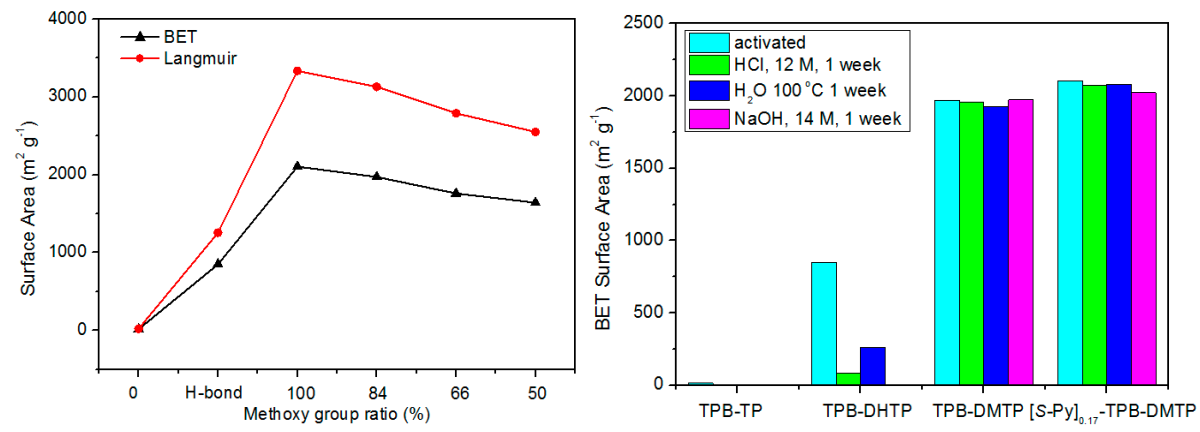

Figure 15. The surface area vs the methoxy group ratio in TPB-DMTP-COF (left). The BET surface areas of TPB-DMTP and (S)-Py-TPB-DMTP COFs use TPB-TP COF and TPB-DHTP COF as control.

Bein and co-workers found that a successive COF layer may nucleate at more than one location during the crystallizaation procedure, and the lateral offset of the growing pattern is more likely to be in various directions, which can induce lattice strain and defect into the crystals of COF materials (Figure 16). To prevent these defects from happening, they developed a synthetic concept to allow consecutive $\mathrm{COF}$ sheets to lock into position during crystal growth, and thus minimize the occurrence 
of stacking faults and dislocations. To prove their concept, they constructed a series of highly crystalline star-shaped, dual-pore 4PE-based COFs by co-condensation of 1,1,2,2-tetrakis(4-aminophenyl)ethane with the linear dialdehyde bridging units 1P, 2P, 3P and TT. They also synthesized a set of hexagonal 3PA- and 3PB-based COFs by combination of the linear dialdehyde bridging units $2 \mathrm{P}$ and TT as control COFs [34]. This approach enabled the achievement of a very high crystallinity for a series of COFs that comprise tri- and tetradentate central building blocks. Bein and co-workers also proposed that the molecular geometry of the central building block in COFs provides a uniquely defined docking site that can lead the attachment of successive COF layers, which will further enhance the crystallinity of the COFs [35]. They chose 1,3,6,8-tetrakis(4-aminophenyl)pyrene as a central building block to synthesize a series of highly crystalline pyrene-based COFs. As shown in Figure 16, the single-crystal structures of their molecular model compounds also revealed different packing preferences for different molecules.

(a)

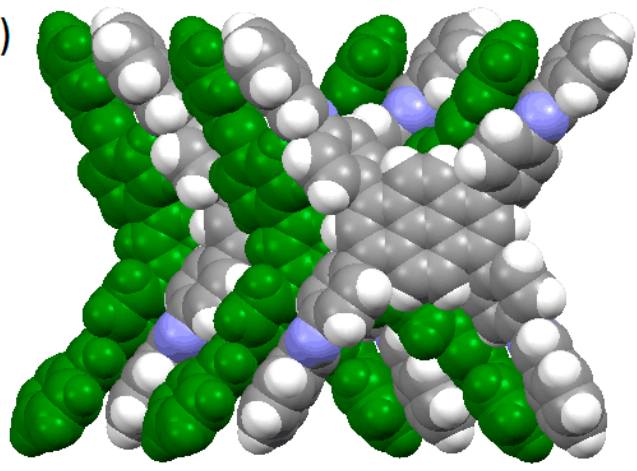

(b)

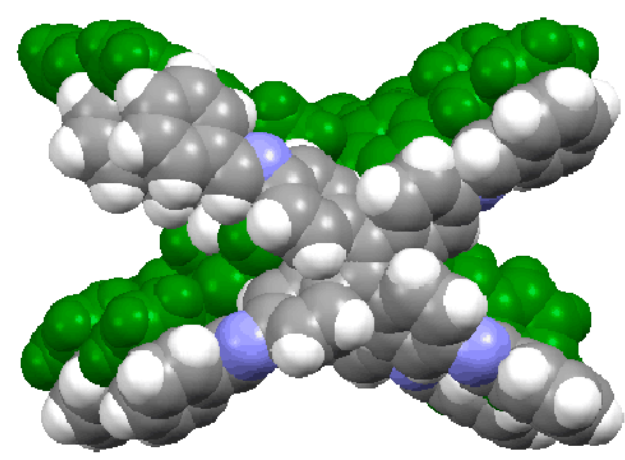

Figure 16. Different packing fashions between building units observed in the discrete model compounds of (a) 4PE-1P COF with 1,1,2,2-tetraphenylethene as the central unit and (b) Py-1P COF with 1,3,6,8-tetrakisphenylpyrene as the central unit, respectively.

To achieve remarkable chemical stability, Banerjee and co-workers explored a new method to enhance the chemical stability of imine COFs by the transformation of combined reversible Schiff base bonds to irreversible $\beta$-ketoenamine bonds (Figure 17) [36]. They synthesized the COF TpPa-1 and TpPa-2 by the Schiff base reactions of 1,3,5-triformylphloroglucinol (Tp) with $p$-phenylenediamine (Pa-1) and 2,5-dimethyl-p-phenylenediamine (Pa-2), respectively. The expected enol-imine $(\mathrm{OH})$ form underwent irreversible proton tautomerization into irreversible keto-enamine form, which confers outstanding stability to boiling water, aqueous acid $(9 \mathrm{~N} \mathrm{HCl})$ and base $(9 \mathrm{~N} \mathrm{NaOH})$. This linkage has also been synthesized by a simple, solvent-free, room-temperature mechanochemical synthetic route [37].The amide-linked COFs show improved chemical stability relative to their imine progenitors, but low degree of crystallinity. Recently, Yaghi and co-workers reported a chemical conversion of imine bond to amide bond inside COFs to improve both the chemical stability (including base and acid stability) and crystallinity (Figure 18). They constructed two layered imine COFs, TPB-TP-COF (1) and $4 \mathrm{PE}-1 \mathrm{P}-\mathrm{COF}$ (2) as starting materials and then converted the imine-linked COFs to the amide-linked COFs, $\left[\mathrm{C}_{6} \mathrm{H}_{3}\left(\mathrm{C}_{6} \mathrm{H}_{4} \mathrm{NH}\right)_{3}\right]_{2}\left[\mathrm{C}_{6} \mathrm{H}_{4}(\mathrm{CO})_{2}\right]_{3}, \mathbf{1}^{\prime}$ and $\left[\left(\mathrm{C}_{6} \mathrm{H}_{4} \mathrm{NH}\right)_{4}\right]\left[\mathrm{C}_{6} \mathrm{H}_{4}(\mathrm{CO})_{2}\right]_{2}$, $\mathbf{2}^{\prime}$ without losing their crystallinity or topology. FT-IR and ${ }^{13} \mathrm{C} C \mathrm{CP}$-MAS NMR spectra indicated the successful conversion of COF 1 and COF 2 to COF $\mathbf{1}^{\prime}$ and COF $\mathbf{2}^{\prime}$. PXRD results demonstrated the high crystal structure of COF $\mathbf{1}^{\prime}$ and COF $\mathbf{2}^{\prime}$. The reduction of BET surface area of COF $\mathbf{1}^{\prime}$ and COF $\mathbf{2}^{\prime}$ compared to COF $\mathbf{1}$ and $\mathrm{COF} 2$ due to the increase of framework mass and decrease of pore volume. This method offers a new pathway to overcome the usual crystallization problem in COF chemistry [38].

\subsubsection{Hydrazone-Linked COFs}

Like the reactions of imine-based COFs, the reaction of benzaldehydes and hydrazide groups could yield hydrazone-linked COFs (Figure 19). Hydrazone-linked COFs show good chemical 
stability relative to imine-based ones due to the hydrogen-bonding interactions between the oxygen atoms in the alkoxyl chains and the hydrogens in -CONH- units. However, the functional monomers available for construction of hydrazone-based COFs are limited. Yaghi and co-workers first condensed a di-functional acylhydrazide (2,5-diethoxyterephthalohydrazide) and trifunctional aldehydes (1,3,5-triformylbenzene or 1,3,5-tris(4-formylphenyl)benzene) to provide two crystalline mesoporous COFs, COF-42 and COF-43 [39].

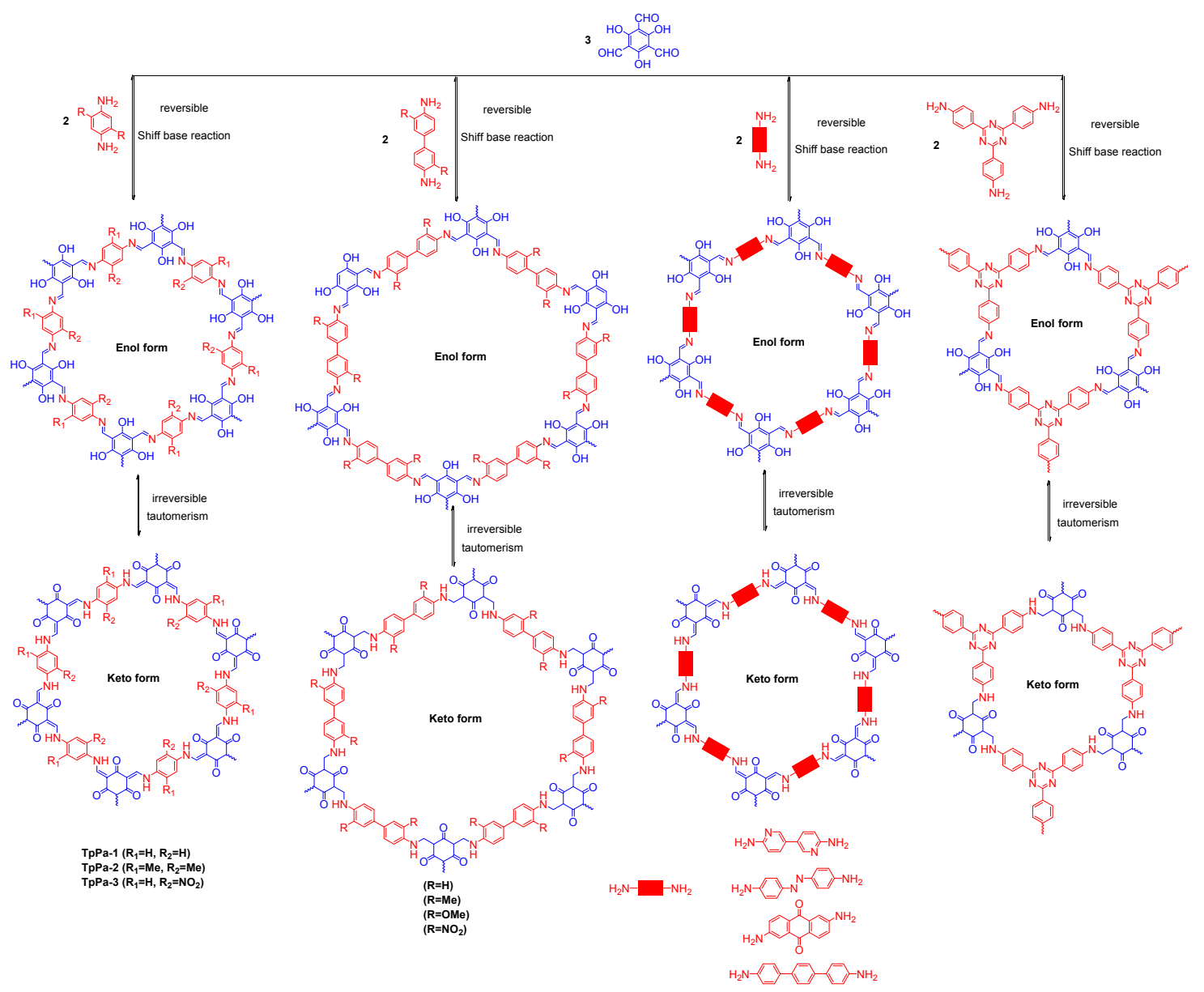

Figure 17. Construction of secondary amine-linked COFs via tautomerism by linking Tp with ditopic or tripic aniline-based linkers.

Dichtel et al. reported the exfoliation of the COF-43 in various solvents to yield few-layer 2D polymers. The obtained exfoliated COF- 43 polymers showed an apparent loss of crystallinity by PXRD, but no apparent changes in its covalent linkages and high aspect ratios [40].

Stegbauer et al. reported a hydrazone-based COF (TFTP-COF) capable of visible-light driven hydrogen generation with $\mathrm{Pt}$ as a proton reduction catalyst (PRC). The COF, constructed by 1,3,5-tris-(4-formyl-phenyl)triazine (TFPT) and 2,5-diethoxy-terephthalohydrazide (DETH) building blocks, shows a layered structure with a honeycomb-type lattice featuring mesopores of $3.8 \mathrm{~nm}$ and gives a BET surface area of $1603 \mathrm{~m}^{2} \cdot \mathrm{g}^{-1}$. When illuminated with visible light, the Pt-doped COF continuously produces hydrogen from water without signs of degradation. This new application of COFs in photocatalysis to open new pathways for heterogeneous photocatalysts [41].

Ding et al. rationally designed a thioether-based hydrazone-linked COF-LZU8, and use it for highly sensitive detection and effective removal of $\mathrm{Hg}^{2+}$. Due to the high stability of the hydrazone linkages, together with the dense distribution of the thioether groups and the straight channels in 
COF-LZU8, the recycling of COF-LZU8 achieved the simultaneous detection and removal of the toxic $\mathrm{Hg}^{2+}[42]$.

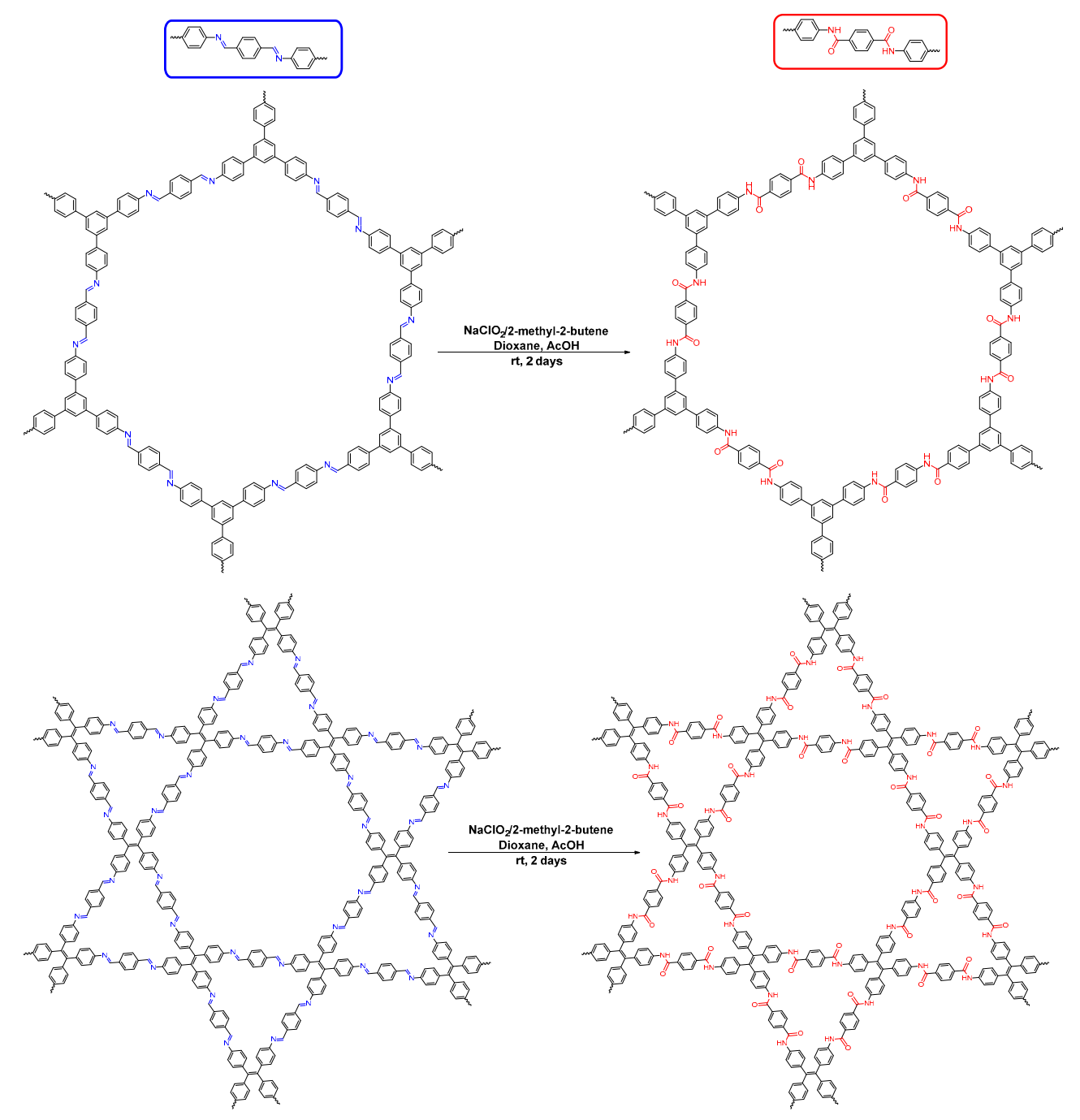

Figure 18. Construction of imide-linked COFs via post-oxidization of imine-linked COFs which bypass the difficulty of crystallization problem for irreversible imide bonds.

\subsubsection{Azine-Linked COFs}

Azines $(-\mathrm{C}=\mathrm{N}-\mathrm{N}=\mathrm{C}-$ ) are formed by condensation of hydrazine with aldehydes (Figure 20). Azine-linked COFs usually have small micropores and are stable in water, acid and base. Dalapati et al. constructed a highly crystalline two-dimensional the Py-Azine COF by condensation of hydrazine with 1,3,6,8-tetrakis(4-formylphenyl)pyrene (TFPPy) under solvothermal conditions [43]. The azines inside of Py-Azine COF serve as Lewis basic sites to bind guest and offers a selective detection of 2,4,6-trinitrophenol (TNP), which exhibits a potential application for chemosensing systems. Li et al. synthesized an azine-linked covalent organic framework, ACOF-1, by condensation of hydrazine hydrate and 1,3,5-triformylbenzene. The high surface areas $\left(1318 \mathrm{~m}^{2} \cdot \mathrm{g}^{-1}\right)$ and small pore size make it useful as a gas storage medium for $\mathrm{CO}_{2}\left(177 \mathrm{mg} \cdot \mathrm{g}^{-1}\right.$ at $273 \mathrm{~K}$ and $\left.1 \mathrm{bar}\right), \mathrm{H}_{2}\left(9.9 \mathrm{mg} \cdot \mathrm{g}^{-1}\right.$ at $273 \mathrm{~K}$ and $1 \mathrm{bar}), \mathrm{CH}_{4}\left(11.5 \mathrm{mg} \cdot \mathrm{g}^{-1}\right.$ at $273 \mathrm{~K}$ and $1 \mathrm{bar}$ [ [44,45]. Smaldone and co-workers developed a novel azine-linked hexaphenylbenzene-based covalent organic framework, HEX-COF 1, which shows excellent sorption capability for carbon dioxide $(20 \mathrm{wt} \%)$ and methane $(2.3 \mathrm{wt} \%)$ at $273 \mathrm{~K}$ and $1 \mathrm{~atm}$ [46]. Lotsch et al. reported a tunable water- and photostable azine COF by hydrazine and 
triphenylarene aldehydes for visible light induced hydrogen generation [47]. Zhang et al. reported an azo-containing COF by introducing a novel azobenzene monomer through the borate ester formation reaction of azobenzene-4,4'-diboronic acid (ABDA) and 2,3,6,7,10,11-hexahydroxytriphenylene (HHTP). The Azo-COF has a hexagonal skeleton and permanent porosity. The azo units in azo-COFs endow the COF material with photoisomerization properties [48].

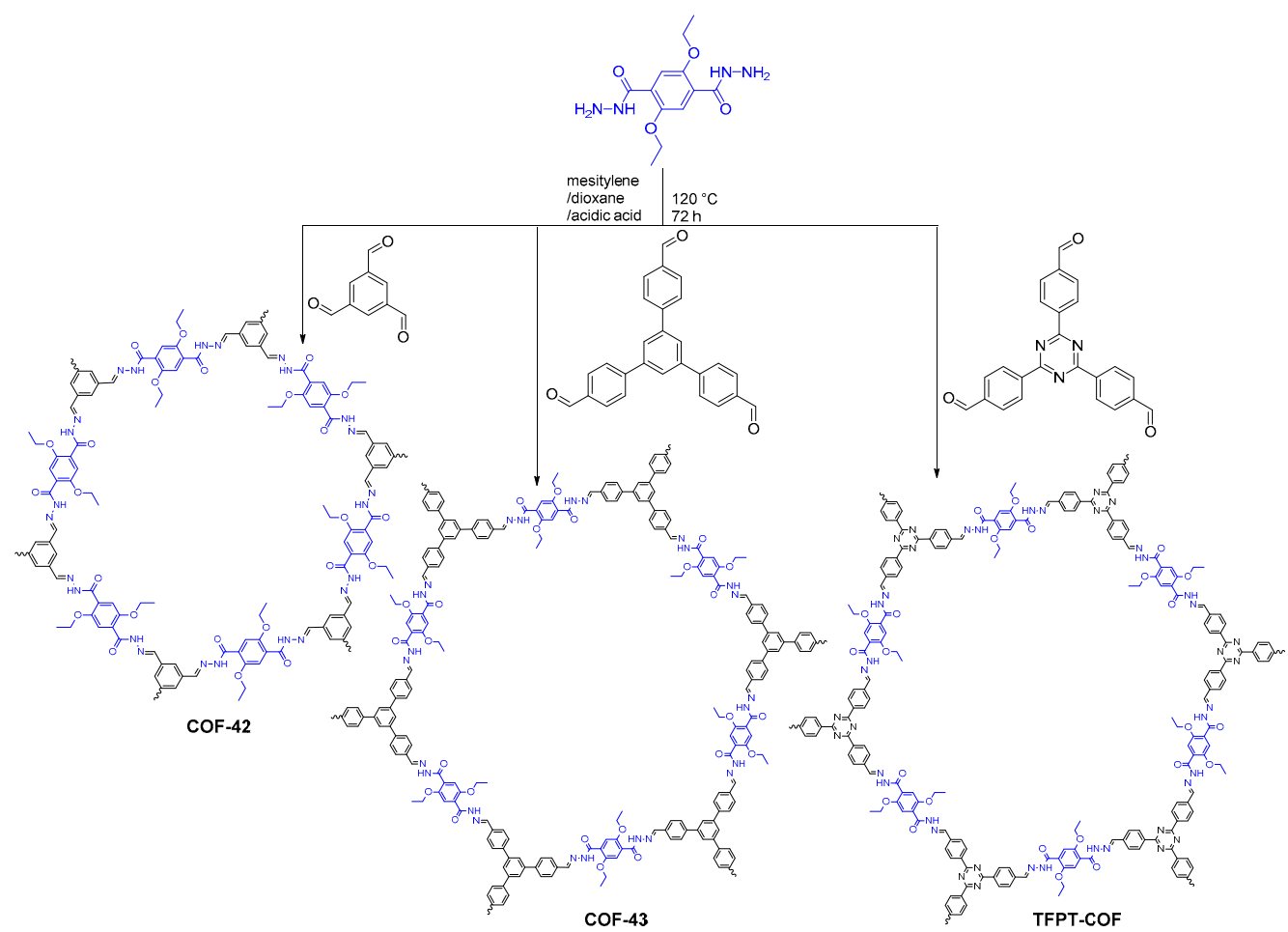

Figure 19. Synthetic reaction of hydrazone-linked COFs (COF-42, COF-43, and TFPT-COF).

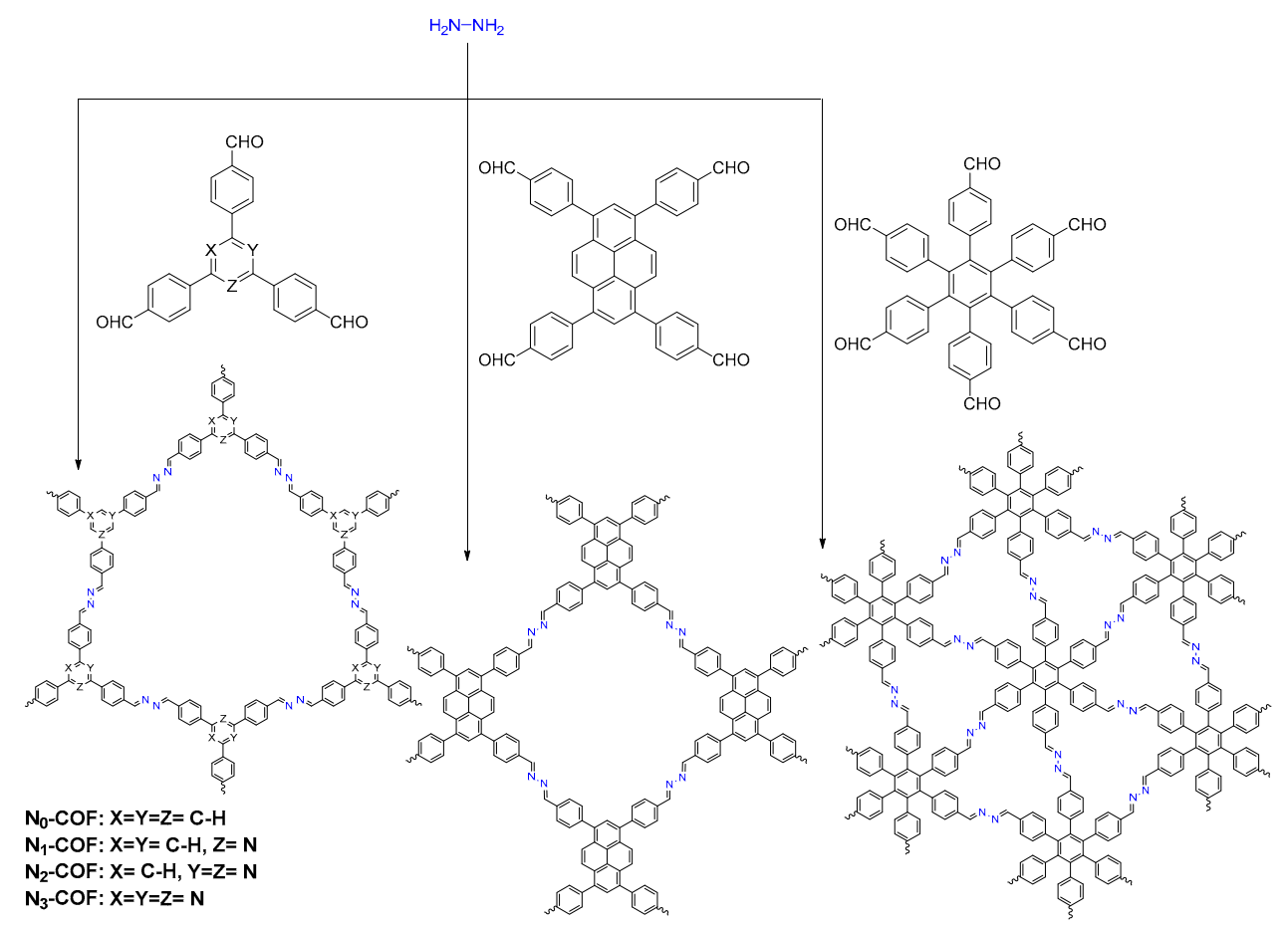

Figure 20. Synthetic reaction of azine-linked COFs (Py-Azine COF, HEX-COF 1, and $\mathrm{N}_{x}-\mathrm{COF}$ ). 


\subsubsection{Squaraine-Linked COF}

The use of squaric acids and amines or hydrazines as building blocks has been developed for the synthesis of squaraine-linked COFs that allow the integration of zwitterion structures into the skeletons. Nagai et al. reported a new reaction based on squaraine for the synthesis of a new type of squaraine-based 2D COF. They used copper(II) 5,10,15,20-tetrakis(4-aminophenyl)porphyrin (TAP-CuP) as a building block to construct a crystalline 2D conjugated COF (CuP-SQ COF) with a tetragonal mesoporous skeleton (Figure 21). The CuP-SQ COF has a BET surface area of $2289 \mathrm{~m}^{2} \cdot \mathrm{g}^{-1}$ with a pore size of $2.1 \mathrm{~nm}$. This research expands the types of COFs. Zhang and their co-workers constructed a novel functional covalent organic framework by the porphyrin building block (meso-tetra-4-hydrazidocarbonylphenyl) porphyrin, THCPP) and squaric acid via a bottom-up approach. The reported covalent porphyrin framework with coordinated manganese (III) ions (Mn-CPF-2) presents promising catalytic properties for the selective oxidation of olefins [49].
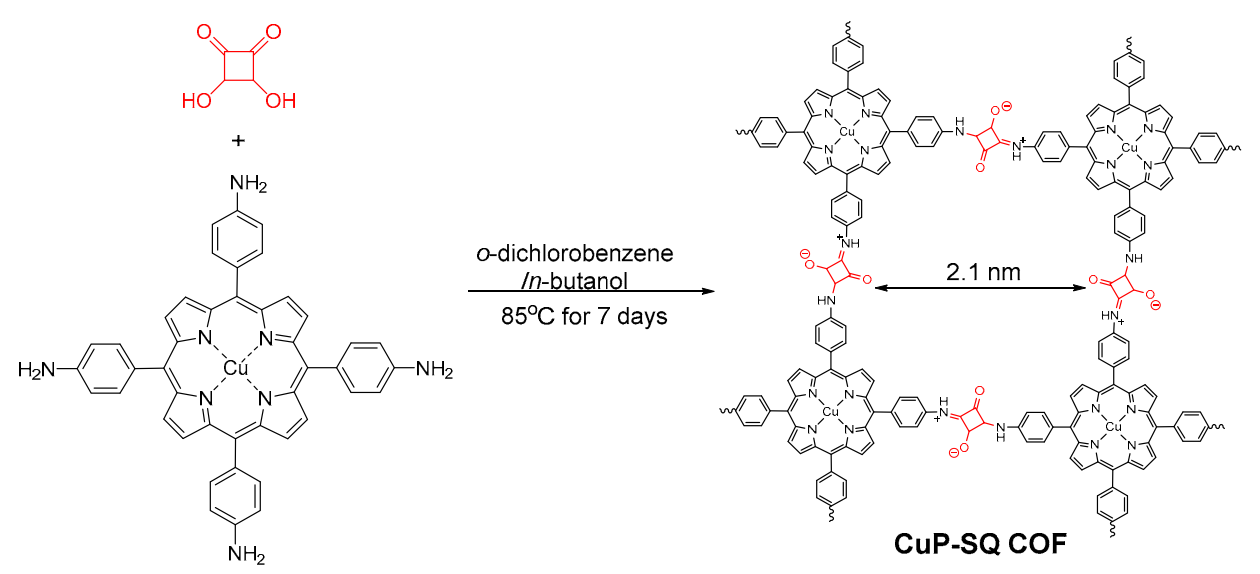

Figure 21. Synthetic reaction of squaraine-linked COFs (CuP-SQ COF).

\subsubsection{Imide-Linked COFs}

Polyimides are formed by condensing primary amines and anhydrides (Figure 22). Yan and co-workers synthesized three-dimensional porous crystalline polyimide covalent organic frameworks (PI-COFs) by choosing tetrahedral building units of different sizes [50].

These PI-COFs show high thermal stability $\left(>450^{\circ} \mathrm{C}\right.$ ) and surface area (up to $2403 \mathrm{~m}^{2} \cdot \mathrm{g}^{-1}$ ), as well as narrow pore size distribution (13 $\AA$ for PI-COF- 4 and $10 \AA$ for PI-COF-5). Due to the high surface area and large pore size, they were the first to be employed in controlled drug delivery, where they show high loadings and good release control for ibuprofen (IBU). These results provide a pathway of COFs for pharmaceutical applications. The Yan group also synthesized a series of polyimide-based COFs with different pore size by changing the length of tri-amines, using the imidization reaction [51]. Among them, the PI-COF-3, synthesized by condensation of PMDA and the extended triamine 1,3,5-tris[4-amino(1,1-biphenyl-4-yl)]benzene (TABPB) has $5.3 \mathrm{~nm}$ wide pores and were loaded with Rhodamine B, a dye probe for biological applications. The dye-doped PI-COF-3 exhibits clear temperature-dependent photoluminescence, which facilitate the development of dye-doped porous materials for applications in temperature-sensing devices. 


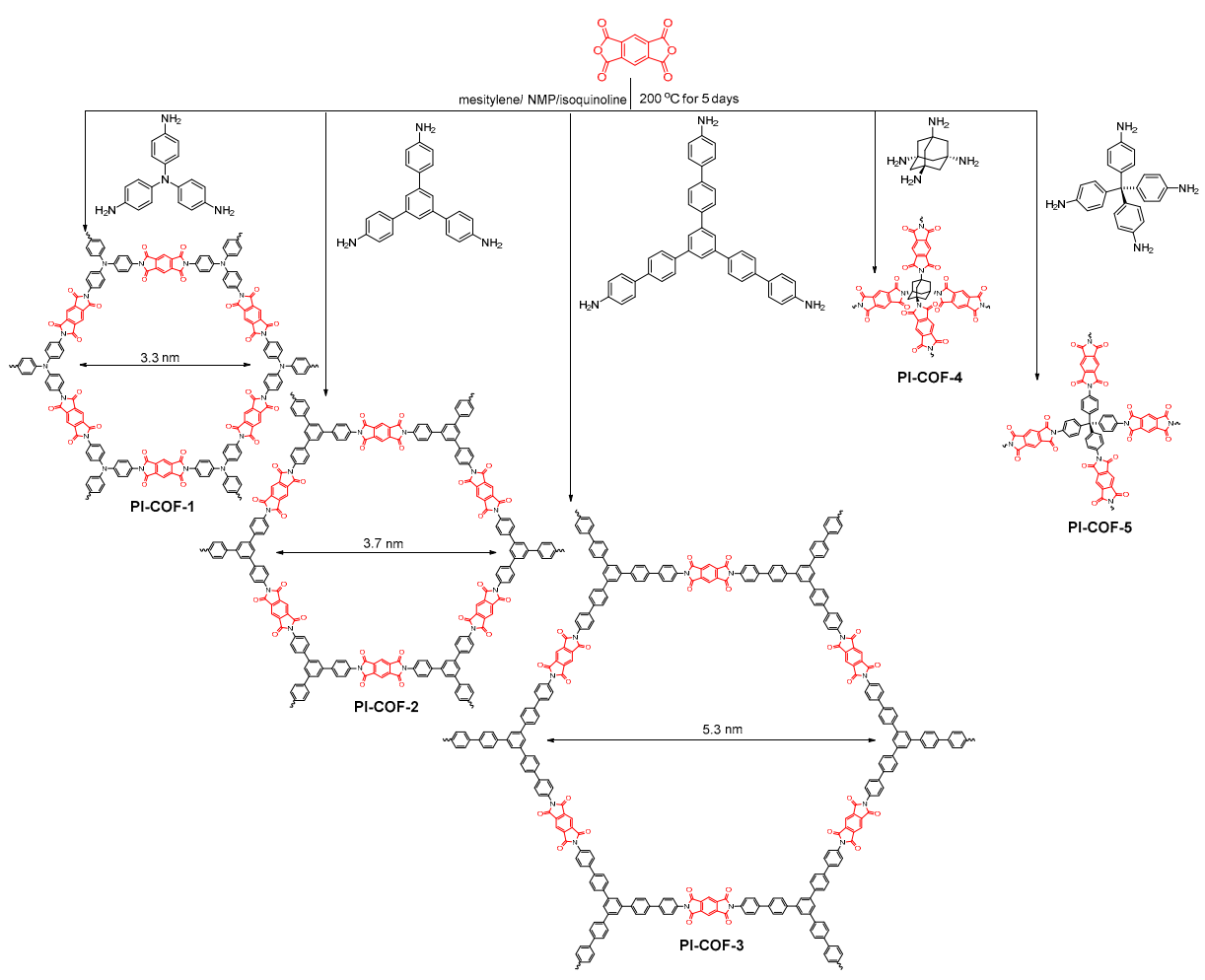

Figure 22. Construction of imide-linked COFs by stitching tritopic or tetratopic polyaniline with benzene-1,2,4,5-tetracarboxylic dianhydride into 2D and 3D networks.

\subsubsection{Phenazine-Linked COFs}

Aza-fused linkages were pursued in making conductive organic materials that have many potential applications in electrochemical devices [52]. However, the great challenge is how to make them highly crystalline. Jiang and co-workers prepared a phenazine-linked COF (CS-COF, Figure 23) through ring-fusing reactions between quinone and amine derivatives catalyzed by acidic forms. They used $\mathrm{C}_{3}$-symmetric triphenylene hexamine (TPHA) and $\mathrm{C}_{2}$-symmetric tertbutylpyrene tetraone (PT) as building blocks to prepare the crystalline phenazine-linked CS-COF under solvothermal conditions. The polygon skeletons of CS-COF are highly $\pi$ conjugated and chemically stable in various solvents [53].

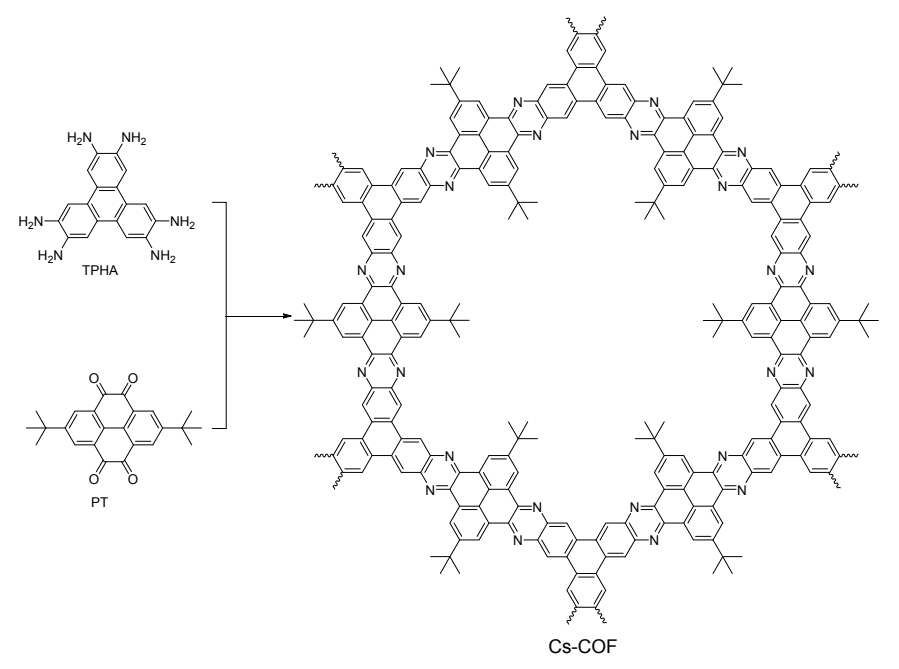

Figure 23. Synthetic reaction of the phenazine-linked COF. 


\subsubsection{Triazine-Linked COFs}

The self-condensation of aromatic nitriles at high temperature in molten salts or at room temperature in the presence of a strong acid catalyst, such as trifluoromethylsulfonic acid, can form crystalline, porous, covalent triazine frameworks (CTFs, Figure 24). The first CTF was reported by Thomas and co-workers in 2008, and is synthesized through the dynamic trimerization of aromatic nitriles (1,3,5-triazines) using a $\mathrm{ZnCl}_{2}$ ionothermal method [54]. CTFs provide high surface area (up to $3000 \mathrm{~m}^{2} \cdot \mathrm{g}^{-1}$ ), low densities, and outstanding thermal/chemical stability. However, CTFs exhibit short-range crystalline order and limited pore size distributions in some cases [55].

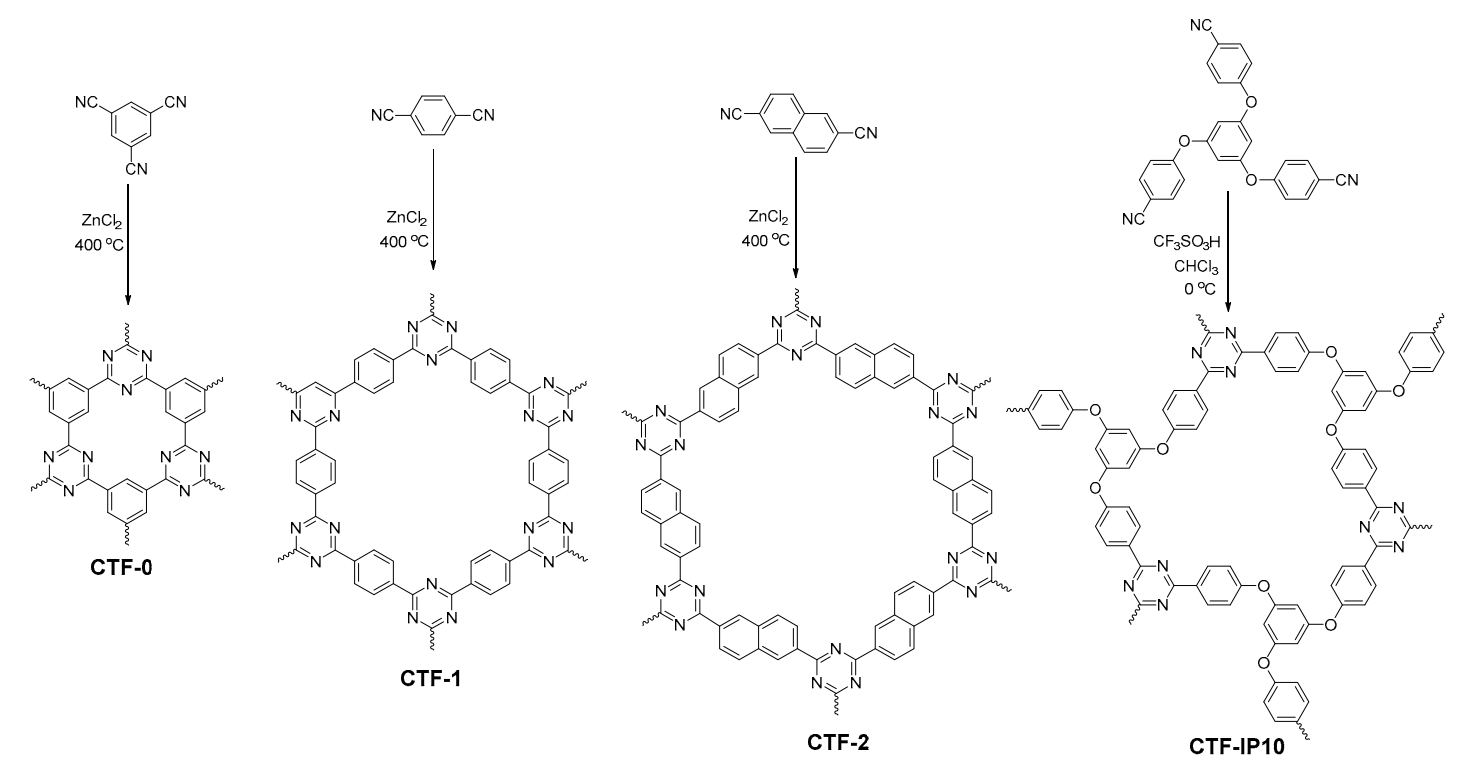

Figure 24. Construction of crystalline covalent triazine frameworks by self-condensation of aromatic nitriles.

Some CTFs were prepared in molten $\mathrm{ZnCl}_{2}$ at high reaction temperatures $\left(400{ }^{\circ} \mathrm{C} \sim 700{ }^{\circ} \mathrm{C}\right)$. Yet these conditions for CTF preparation obtained less crystalline CTFs of limited functionality, and only from 1,4-dicyanobenzene and 2,6-dicyanonaphthalene [56]. Then, Cooper et al. [57] developed a microwave-assisted, acid-catalyzed method to synthesize triazine networks with fewer discolored impurities (P1M-P6M). Their structural and synthesized tunability developed their specific applications, such as gas storage, gas separation, catalyst support, and organic dye separation.

Thomas et al. synthesized a covalent triazine framework (CTF-0) by trimerization of 1,3,5-tricyanobenzene in molten $\mathrm{ZnCl}_{2}$ [58]. This material showed high $\mathrm{CO}_{2}$ uptakes and good catalytic activity for $\mathrm{CO}_{2}$ cycloaddition. The high surface area and robust porous structures of the organic framework were explored for highly selective gas capture.

Ghosh et al. explored a strategy to obtain a porous covalent triazine framework (CTF-IP-10) with bimodal functionality by acid-catalysed room temperature reaction of a tricyanomonomer (PCN-M1) in $\mathrm{CHCl}_{3}$ [59]. The obtained CTF-IP 10 consists both an electron-deficient central triazine core and electron-rich aromatic building blocks.

\subsection{Hetero Linkages in One COF Structure}

To improve the functionality and stability of imine-based COF structures, design and construction of COFs connected by two or more types of covalent bonds based on orthogonal reactions were developed.

First the Zhao group constructed a 2D COF (NTU-COF-1, Figure 25) by the formation of imine groups and $\mathrm{B}_{3} \mathrm{O}_{3}$ boroxine rings, involving the utilization of two building blocks: 4-formyl-phenylboronic acid 
(FPBA) and 1,3,5-tris(4-aminophenyl)-benzene (TAPB). They also constructed a ternary COF (NTU-COF-2, Figure 25) through the formation of imine groups and $\mathrm{C}_{2} \mathrm{O}_{2} \mathrm{~B}$ boronate rings from FPBA, TAPB and HHTP, which involved the formation of two types of covalent bonds. The NTU-COF-2 possesses high BET surface areas of $1619 \mathrm{~m}^{2} \cdot \mathrm{g}^{-1}$ and mesopores with a diameter of $2.6 \mathrm{~nm}$. The design and construction of COFs by this orthogonal reaction strategy is a promising approach in rational COF development [60].

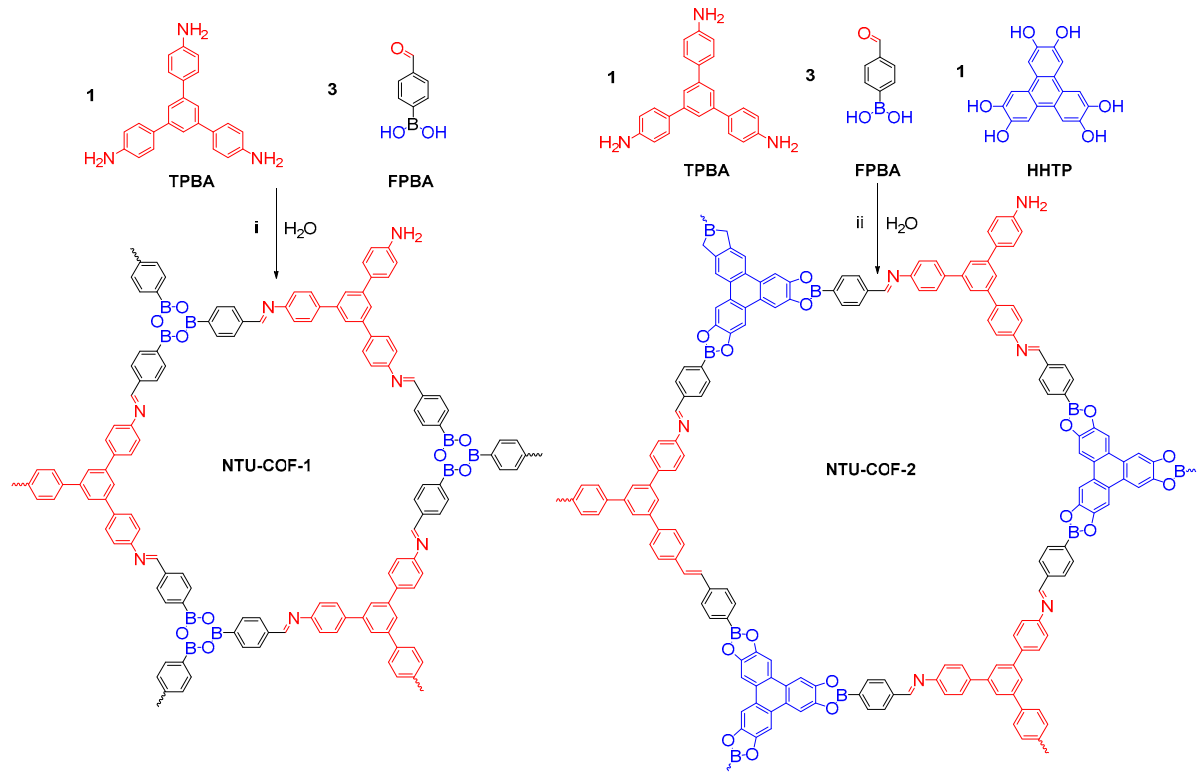

Figure 25. Syntheses of (i) NTU-COF-1 and (ii) NTU-COF-2 by two types of covalent bonds from orthogonal reactions.

The Qiu group synthesized two extended 3D COFs (DL-COF-1, DL-COF-2) with dual linkages, imine groups and $\mathrm{B}_{3} \mathrm{O}_{3}$ boroxine rings (Figure 26), involving the utilization of two 1,3,5,7-tetraaminoadamantane (TAA) and 4-formylphenylboronic acid (FPBA) or 2-fluoro-4-formylphenylboronic acid (FFPBA) building blocks [61]. The obtained DL-COF-1 and DL-COF-2 showed high BET surface areas of $2259 \mathrm{~m}^{2} \cdot \mathrm{g}^{-1}$ and $2071 \mathrm{~m}^{2} \cdot \mathrm{g}^{-1}$, respectively.

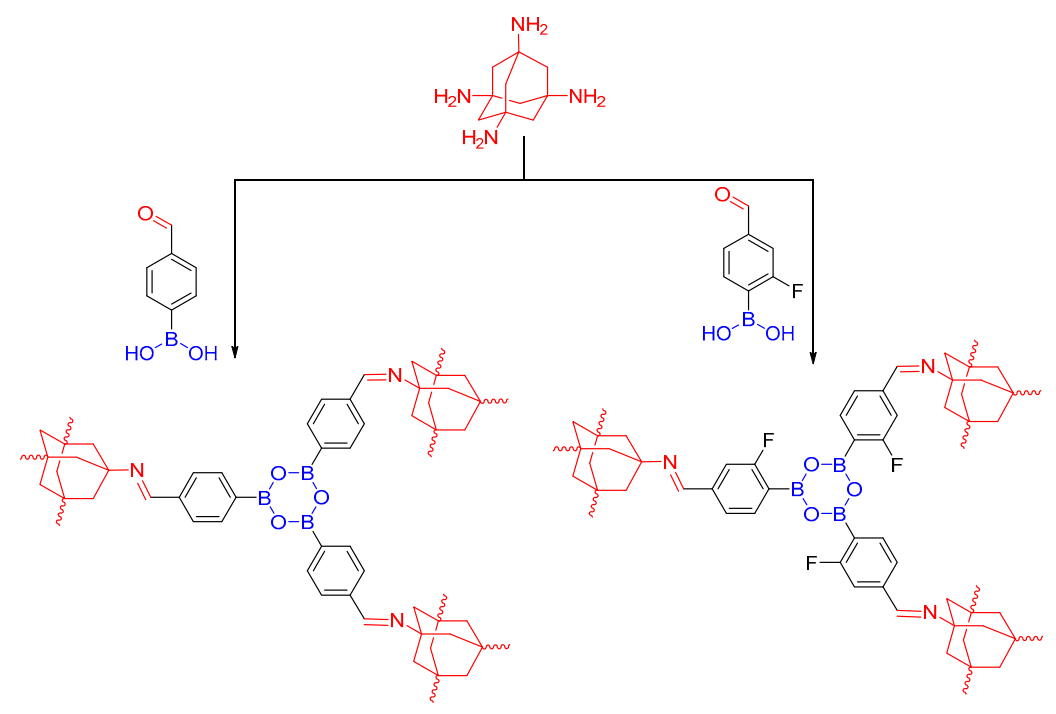

Figure 26. Syntheses 3D COFs of (i) DL-COF-1 and (ii) DL-COF-2 by two types of covalent bonds from orthogonal reactions. 


\section{Gas Storage for Clean Energy Applications}

COFs have been touted as a new class of porous crystalline materials for gas storage applications due to their high inherent surface area, very low densities (i.e., being made of the elements $\mathrm{H}, \mathrm{C}, \mathrm{N}, \mathrm{B}$, $\mathrm{O}, \mathrm{Si}$ ), high stability (formation of covalent bonds), and tunable pore dimensions (from ultramicropores to mesopores). The gas storage capacity of COFs for hydrogen, methane, and carbon dioxide, has attracted increasing interest in the past decades [62].

\subsection{Hydrogen Storage}

The demand for the reduction of air pollution has led to the search of new and clean energy sources. Hydrogen gas storage is one of the bottleneck for the realization of an energy economy based on hydrogen's high chemical abundance, being a clean energy carrier, and high chemical energy density. The demand of the US Department of Energy (DOE) for hydrogen storage is $5.5 \mathrm{wt} \%$ in gravimetric capacity, and $40 \mathrm{~kg} \cdot \mathrm{m}^{-3}$ of volumetric capacity at operating temperature of $233-333 \mathrm{~K}$ with a pressure of $100 \mathrm{~atm}$ by the year 2017. Recently, a large number of porous materials have been explored for the application of hydrogen storage. However, none of the candidate materials developed so far has satisfied the DOE target. COF materials, as new crystal porous materials, have also attracted increasing interest in hydrogen storage applications.

Figure 27 and Table 1 show the capabilities of hydrogen storage reported for different COF materials. The plot of hydrogen uptake against surface area and pore volume for the selected COFs at

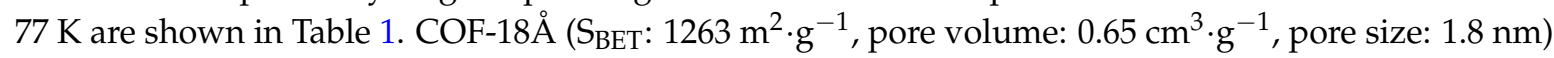
showed the highest hydrogen uptake at low pressure ( 1 bar and $77 \mathrm{~K}$ ) among the similar 2D COFs with

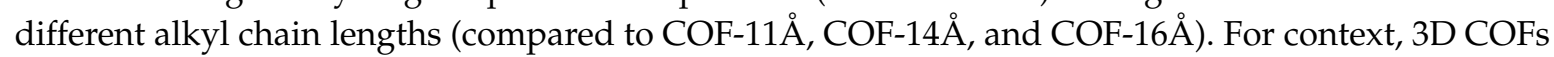
(COF-102 and COF-103) show much higher hydrogen storage capacities due to the higher surface areas and lower densities, compared to 2D COFs. Among the different COF materials for $\mathrm{H}_{2}$ uptake, the one with the largest storage capacity is 3D COF-102 (Table $1, \mathrm{~S}_{\mathrm{BET}}: 3620 \mathrm{~m}^{2} \cdot \mathrm{g}^{-1}$, pore volume: $1.55 \mathrm{~cm}^{3} \cdot \mathrm{g}^{-1}$, pore size: $1.2 \mathrm{~nm}$ ), which uptakes $72 \mathrm{mg}$ per gram at 1 bar and $77 \mathrm{~K}$. This capacity is comparable to those of MOF-177 $\left(75 \mathrm{mg} \cdot \mathrm{g}^{-1}, \mathrm{~S}_{\mathrm{BET}}: 4500 \mathrm{~m}^{2} \cdot \mathrm{g}^{-1}\right)$, MOF-5 $\left(76 \mathrm{mg} \cdot \mathrm{g}^{-1}, \mathrm{~S}_{\mathrm{BET}}: 3800 \mathrm{~m}^{2} \cdot \mathrm{g}^{-1}\right)$, and the porous aromatic framework PAF-1 $\left(75 \mathrm{mg} \cdot \mathrm{g}^{-1}, \mathrm{~S}_{\mathrm{BET}}: 5600 \mathrm{~m}^{2} \cdot \mathrm{g}^{-1}\right)$. These capacities significantly demonstrate the potential of COFs for use as hydrogen storage materials.

Table 1. BET surface area, porosity parameters and $\mathrm{H}_{2}$ capture of selected COFs.

\begin{tabular}{|c|c|c|c|c|c|}
\hline COF Materials & BET Surface Area $\left(\mathrm{m}^{2} \cdot \mathrm{g}^{-1}\right)$ & Pore Size (Å) & Pore Volume $\left(V_{p}, \mathrm{~cm}^{3} \cdot \mathrm{g}^{-1}\right)$ & $\mathrm{H}_{2}$ Uptake (wt \%) & Ref. \\
\hline COF-5 & 1670 & 27 & 1.07 & 3.58 & \\
\hline COF-6 & 750 & 6.4 & 0.32 & 2.26 & \\
\hline COF-10 & 1760 & 34.1 & 1.44 & 3.92 & \\
\hline COF-102 & 3620 & 11.5 & 1.55 & 7.24 & \\
\hline COF-103 & 3530 & 12.5 & 1.54 & 7.05 & \\
\hline COF-14Å & 805 & 14 & 0.41 & $1.23(1 \mathrm{bar})$ & \\
\hline COF-16 & 753 & 16 & 0.39 & 1.4 (1 bar) & \\
\hline 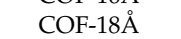 & 1263 & 18 & 0.69 & 1.55 (1 bar) & \\
\hline CTC-COF & 1710 & 22.6 & 1.03 & 1.12 (1.05 bar) & \\
\hline dualpore-COF & 1771 & $7.3,25.2$ & $3.189\left(\mathrm{P} / \mathrm{P}^{\circ} 0.99\right)$ & 1.37 (1 bar) & \\
\hline
\end{tabular}

From the summarized $\mathrm{H}_{2}$ storage results in Figure 27, we find that the high-pressure $\mathrm{H}_{2}$ adsorption of $\mathrm{COFs}$ with higher surface areas and larger pore possess higher hydrogen uptake capacities when measured under the same conditions. Meanwhile, the COFs with higher pore volume show higher hydrogen uptake. These results demonstrate that the high-pressure $\mathrm{H}_{2}$ uptake capacities are dominantly determined by pore volumes and $\mathrm{S}_{\mathrm{BET}}$ of COFs. The $\mathrm{H}_{2}$ uptake capacity at $77 \mathrm{~K}$ and 1 bar increased in the order of COF- $11 \AA \AA$ COF- $14 \AA<$ COF- $16 \AA<$ COF- $18 \AA$, revealing that the low-pressure $\mathrm{H}_{2}$ uptake also depends on the pore size of COFs. 

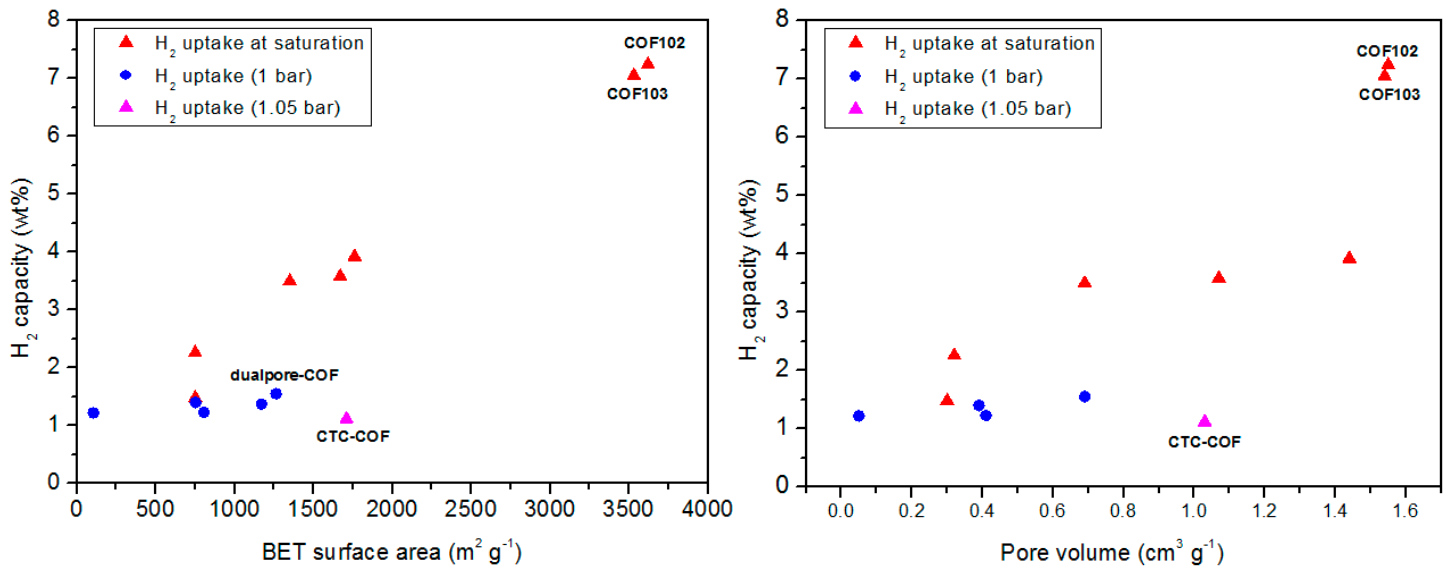

Figure 27. Plot of $\mathrm{H}_{2}$ uptake against BET surface area (left) and pore volume (right) for the selected $\mathrm{COFs}$ at $77 \mathrm{~K}$.

However, the hydrogen storage of COFs at ambient pressure and temperature is still low and far from meeting the DOE requirements. Adsorption simulations of the COFs show that doping with charged species can affect their capacity. Theoretical studies $[63,64]$ show that metal-doped COFs can enhance the hydrogen storage at a temperature range of 273 to $298 \mathrm{~K}$. Recently, scientists have theoretically predicted that lithium doped COFs would exhibit improved hydrogen storage capacity due to the increased binding energy between the $\mathrm{H}_{2}$ and the $\mathrm{Li}$ atom. These simulation studies predict that the hydrogen uptakes for Li-doped COF-105 and COF-108 (6.84 and $6.73 \mathrm{wt} \%$, respectively, at $298 \mathrm{~K}$ and 100 bar) excelled beyond the non-doped COFs and the previous reported MOF materials. Although the experimental and theoretical results indicate that COFs are promising candidates for hydrogen storage, the practical and industrial use of COF materials toward the DOE target for hydrogen uptakes is still far away.

\subsection{Methane Storage}

Methane, as an abundant and inexpensive gas, stands out as a potential vehicular fuel to replace conventional fossil fuels. According to the US DOE requirement, the current target for methane storage is $350 \mathrm{~cm}^{3}{ }_{\text {STP }} \mathrm{cm}^{-3}$ adsorbent $(v / v)$ and $0.5 \mathrm{~g}_{\mathrm{CH} 4} \mathrm{~g}_{\text {adsorbent }}{ }^{-1}\left(699 \mathrm{~cm}^{3}\right.$ STP $\left.^{-1}\right)$ at 35 bar and $298 \mathrm{~K}$ (STP: standard temperature and pressure). From Table 2, the 2D COF, COF-1 shows $\mathrm{CH}_{4}$ uptake of $40 \mathrm{mg} \cdot \mathrm{g}^{-1}$ at $298 \mathrm{~K}, 35$ bar. However, upon increasing the pressure further, no increase of methane adsorption is observed due to the small pores in COF-1. The pores in COF-1 are quickly occupied by $\mathrm{CH}_{4}$ molecules at low pressures and higher pressures may not compact the gas any more. The largest pore, 3D COFs, COF-102 shows the highest observed $\mathrm{CH}_{4}$ storage capacity $\left(187 \mathrm{mg} \cdot \mathrm{g}^{-1}\right)$. COF-103 also possesses a high methane capacity of $175 \mathrm{mg} \cdot \mathrm{g}^{-1}$. These values are comparable to the highest methane uptake for MOFs (MOF-210; $220 \mathrm{mg} \cdot \mathrm{g}^{-1}$ ). Theoretical simulation studies indicate that lithium doped COFs could significantly strengthen the binding between methane and $\mathrm{Li}^{+}$cations, which will further improve the methane capacity of COFs. The results show that the methane storage of lithium doped COFs are double at $298 \mathrm{~K}$ and low pressures (<50 bar), compared to the non-doped COFs.

The abovementioned studies indicate the potential application of COFs for methane storage at ambient temperatures and 35 bar. However, the practical use of COF materials to meet the requirement of DOE target for is a big challenge. 
Table 2. BET surface area, porosity parameters and $\mathrm{CH}_{4}$ capture of selected COFs.

\begin{tabular}{|c|c|c|c|c|c|}
\hline COF Materials & BET Surface Area $\left(\mathrm{m}^{2} \cdot \mathrm{g}^{-1}\right)$ & Pore Size $(\AA)$ & Pore Volume $\left(V_{p}, \mathrm{~cm}^{3} \cdot \mathrm{g}^{-1}\right)$ & $\mathrm{CH}_{4}$ Uptake $\left(\mathrm{mg} \cdot \mathrm{g}^{-1}\right)^{a}$ & Ref. \\
\hline COF-1 & 750 & 9 & 0.3 & 40 & [62] \\
\hline COF-5 & 1670 & 27 & 1.07 & 89 & \\
\hline COF-6 & 750 & 6.4 & 0.32 & 65 & \\
\hline COF-8 & 1350 & 18.7 & 0.69 & 87 & \\
\hline COF-10 & 1760 & 34.1 & 1.44 & 80 & \\
\hline COF-102 & 3620 & 11.5 & 1.55 & 187 & \\
\hline COF-103 & 3530 & 12.5 & 1.54 & 175 & \\
\hline ILCOF-1 & 2723 & 23 & 1.21 & $129\left(\mathrm{~L} \cdot \mathrm{L}^{-1}\right)$ & \\
\hline
\end{tabular}

${ }^{a} \mathrm{CH}_{4}$ uptake at 35 bar and $298 \mathrm{~K}$.

\section{3. $\mathrm{CO}_{2}$ Capture}

The rise of carbon dioxide is one of the global issues, which is causing global warming, rising sea levels, and the increasing acidity of the oceans. The development of effective strategies for capturing $\mathrm{CO}_{2}$ has attracted broad interest. Among the current strategies, capture of $\mathrm{CO}_{2}$ by crystal porous materials is energetically efficient and technically feasible. Yaghi and co-workers first studied a series of 2D COFs with 1D micropores (COF-1 and COF-6), 2D COFs with 1D mesopores (COF-5, COF-8 and COF-10), and 3D COFs with 3D medium sized pores (COF-102 and COF-103) on application of $\mathrm{CO}_{2}$ capture in 2009. From Table 3, we can observe that the storage of $\mathrm{CO}_{2}$ at $298 \mathrm{~K}$ and 55 bar, follow the order of COF-102 > COF-103 > COF-10 > COF-5 > COF-8 > COF- $6>$ COF-1, which indicates that the high-pressure $\mathrm{CO}_{2}$ capture are highly related to the pore volumes and $\mathrm{SA}_{\mathrm{BET}}$ of COFs.

Table 3. BET surface area, porosity parameters and $\mathrm{CO}_{2}$ capture of selected COFs.

\begin{tabular}{|c|c|c|c|c|c|c|}
\hline \multirow{2}{*}{ COF Materials } & \multirow{2}{*}{ BET Surface Area $\left(\mathrm{m}^{2} \cdot \mathrm{g}^{-1}\right)$} & \multirow{2}{*}{ Pore Size (nm) } & \multirow{2}{*}{ Pore Volume $\left(V_{\mathrm{p}}, \mathrm{cm}^{3} \cdot \mathrm{g}^{-1}\right)$} & \multicolumn{2}{|c|}{$\mathrm{CO}_{2}$ Uptake $\left(\mathrm{cm}^{3} \cdot \mathrm{g}^{-1}\right)$} & \multirow{2}{*}{ Ref. } \\
\hline & & & & Low $^{a}$ & High $^{b}$ & \\
\hline COF-1 & 750 & 0.9 & 0.3 & 51 & 117 & [39] \\
\hline COF-5 & 1670 & 2.7 & 1.07 & 31 & 443 & \\
\hline COF-6 & 750 & 0.64 & 0.32 & 85 & 158 & \\
\hline COF-8 & 1350 & 1.87 & 0.69 & 33 & 321 & \\
\hline COF-10 & 1760 & 3.41 & 1.44 & 27 & 514 & \\
\hline COF-102 & 3620 & 1.15 & 1.55 & 34 & 611 & \\
\hline COF-103 & 3530 & 1.25 & 1.54 & 38 & 606 & \\
\hline FCTF-1 & 662 & $0.46,0.54$ & - & 105 & & [65] \\
\hline FCTF-1-600 & 1535 & $0.46,0.59$ & - & 124 & & \\
\hline CTF-1 & 746 & 0.54 & & 55 & & \\
\hline CTF-1-600 & 1553 & - & & 80 & & \\
\hline$[\mathrm{HO}]_{25 \%}-\mathrm{H}_{2} \mathrm{P}-\mathrm{COF}$ & 1054 & 2.5 & & 27 & & [66] \\
\hline$[\mathrm{HO}]_{50 \%}-\mathrm{H}_{2} \mathrm{P}-\mathrm{COF}$ & 1089 & 2.5 & & 23 & & \\
\hline$[\mathrm{HO}]_{75 \%}-\mathrm{H}_{2} \mathrm{P}-\mathrm{COF}$ & 1153 & 2.5 & & 26 & & \\
\hline$[\mathrm{HO}]_{100 \%}-\mathrm{H}_{2} \mathrm{P}-\mathrm{COF}$ & 1284 & 2.5 & & 32 & & \\
\hline$\left[\mathrm{HO}_{2} \mathrm{C}_{25 \%} \% \mathrm{H}_{2} \mathrm{P}-\mathrm{COF}\right.$ & 786 & 2.2 & & 49 & & \\
\hline$\left[\mathrm{HO}_{2} \mathrm{C}\right]_{50 \%}-\mathrm{H}_{2} \mathrm{P}-\mathrm{COF}$ & 673 & 1.9 & & 68 & & \\
\hline$\left[\mathrm{HO}_{2} \mathrm{C}_{75 \%}-\mathrm{H}_{2} \mathrm{P}-\mathrm{COF}\right.$ & 482 & 1.7 & & 80 & & \\
\hline$\left[\mathrm{HO}_{2} \mathrm{C}\right]_{100 \%}-\mathrm{H}_{2} \mathrm{P}-\mathrm{COF}$ & 364 & 1.4 & & 89 & & \\
\hline$[\mathrm{CH} \equiv \mathrm{C}]_{0}-\mathrm{H}_{2} \mathrm{P}-\mathrm{COF}$ & 1474 & 2.5 & 0.75 & 37 & & [67] \\
\hline$\left[\mathrm{CH} \equiv \mathrm{C}_{25}-\mathrm{H}_{2} \mathrm{P}-\mathrm{COF}\right.$ & 1413 & 2.3 & 0.71 & 27 & & \\
\hline$[\mathrm{CH} \equiv \mathrm{C}]_{50}-\mathrm{H}_{2} \mathrm{P}-\mathrm{COF}$ & 962 & 2.1 & 0.57 & 24 & & \\
\hline$[\mathrm{CH} \equiv]_{75}-\mathrm{H}_{2} \mathrm{P}-\mathrm{COF}$ & 683 & 1.9 & 0.42 & 22 & & \\
\hline$[\mathrm{CH} \equiv \mathrm{C}]_{100}-\mathrm{H}_{2} \mathrm{P}-\mathrm{COF}$ & 462 & 1.6 & 0.28 & 20 & & \\
\hline$[\mathrm{Et}]_{25}-\mathrm{H}_{2} \mathrm{P}-\mathrm{COF}$ & 1326 & 2.2 & 0.55 & 28 & & \\
\hline$[\mathrm{Et}]_{50}-\mathrm{H}_{2} \mathrm{P}-\mathrm{COF}$ & 821 & 1.9 & 0.48 & 23 & & \\
\hline$\left[\mathrm{Et}_{75}-\mathrm{H}_{2} \mathrm{P}-\mathrm{COF}\right.$ & 485 & 1.6 & 0.34 & 21 & & \\
\hline$[\mathrm{Et}]_{100}-\mathrm{H}_{2} \mathrm{P}-\mathrm{COF}$ & 187 & 1.5 & 0.18 & 19 & & \\
\hline$[\mathrm{MeOAc}]_{25}-\mathrm{H}_{2} \mathrm{P}-\mathrm{COF}$ & 1238 & 2.1 & 0.51 & 43 & & \\
\hline$[\mathrm{MeOAc}]_{50}-\mathrm{H}_{2} \mathrm{P}-\mathrm{COF}$ & 754 & 1.8 & 0.42 & 45 & & \\
\hline$[\mathrm{MeOAc}]_{75}-\mathrm{H}_{2} \mathrm{P}-\mathrm{COF}$ & 472 & 1.5 & 0.31 & 42 & & \\
\hline$[\mathrm{MeOAc}]_{100}-\mathrm{H}_{2} \mathrm{P}-\mathrm{COF}$ & 156 & 1.1 & 0.14 & 33 & & \\
\hline$[\mathrm{AcOH}]_{25}-\mathrm{H}_{2} \mathrm{P}-\mathrm{COF}$ & 1252 & 2.2 & 0.52 & 48 & & \\
\hline$[\mathrm{AcOH}]_{50}-\mathrm{H}_{2} \mathrm{P}-\mathrm{COF}$ & 866 & 1.8 & 0.45 & 60 & & \\
\hline$[\mathrm{AcOH}]_{75}-\mathrm{H}_{2} \mathrm{P}-\mathrm{COF}$ & 402 & 1.5 & 0.32 & 55 & & \\
\hline$[\mathrm{AcOH}]_{100}-\mathrm{H}_{2} \mathrm{P}-\mathrm{COF}$ & 186 & 1.3 & 0.18 & 49 & & \\
\hline$[\mathrm{EtOH}]_{25}-\mathrm{H}_{2} \mathrm{P}-\mathrm{COF}$ & 1248 & 2.2 & 0.56 & 47 & & \\
\hline$[\mathrm{EtOH}]_{50}-\mathrm{H}_{2} \mathrm{P}-\mathrm{COF}$ & 784 & 1.9 & 0.43 & 63 & & \\
\hline$[\mathrm{EtOH}]_{75}-\mathrm{H}_{2} \mathrm{P}-\mathrm{COF}$ & 486 & 1.6 & 0.36 & 60 & & \\
\hline$[\mathrm{EtOH}]_{100}-\mathrm{H}_{2} \mathrm{P}-\mathrm{COF}$ & 214 & 1.4 & 0.19 & 43 & & \\
\hline$\left[\mathrm{EtNH}_{2}\right]_{25}-\mathrm{H}_{2} \mathrm{P}-\mathrm{COF}$ & 1402 & 2.2 & 0.58 & 59 & & \\
\hline$\left[\mathrm{EtNH}_{2}\right]_{50}-\mathrm{H}_{2} \mathrm{P}-\mathrm{COF}$ & 1044 & 1.9 & 0.5 & 80 & & \\
\hline$\left[\mathrm{EtNH}_{2}\right]_{75}-\mathrm{H}_{2} \mathrm{P}-\mathrm{COF}$ & 568 & 1.6 & 0.36 & 68 & & \\
\hline$\left[\mathrm{EtNH}_{2}\right]_{100}-\mathrm{H}_{2} \mathrm{P}-\mathrm{COF}$ & 382 & 1.3 & 0.21 & 49 & & \\
\hline
\end{tabular}

${ }^{a} \mathrm{CO}_{2}$ uptake was measured at 1 bar and $273 \mathrm{~K}^{b} \mathrm{CO}_{2}$ uptake was measured at 55 bar and $298 \mathrm{~K}$. 
Functional groups inside the channel walls of $\mathrm{COF}$ materials can enhance the $\mathrm{CO}_{2}$ adsorption and separation. Fluorinated alkanes exhibit an extraordinary affinity to $\mathrm{CO}_{2}$ molecules. Han et al. reported a fluorinated triazine-based COF (FCFT-1 and FCTF-1-600) for $\mathrm{CO}_{2}$ capture by using tetrafluoroterephthalonitrile as a monomer [65]. The triazine-based COFs haveN-rich frameworks, which are favored for $\mathrm{CO}_{2}$ adsorption, meanwhile, the high electronegativity of $\mathrm{F}$ enhances $\mathrm{CO}_{2}$ electrostatic interaction. The $\mathrm{CO}_{2}$ adsorption of these triazine-based COF materials at $273 \mathrm{~K}$ and 1 bar were as follows, FCTF-1-600 $\left(124 \mathrm{~cm}^{3} \cdot \mathrm{g}^{-1}\right)>$ FCTF- $1\left(105 \mathrm{~cm}^{3} \cdot \mathrm{g}^{-1}\right)>$ CTF-1-600 $\left(86 \mathrm{~cm}^{3} \cdot \mathrm{g}^{-1}\right)$ $>$ CTF-1 $\left(55 \mathrm{~cm}^{3} \cdot \mathrm{g}^{-1}\right)$. These results demonstrate that the fluorizated COF materials exhibit much better $\mathrm{CO}_{2}$ uptake under low pressure. Jiang et al. converted a conventional hydroxyl group modified 2D COF $\left([\mathrm{HO}]_{\mathrm{x} \%}-\mathrm{H}_{2} \mathrm{P}-\mathrm{COFs}\right)$ into an outstanding carbondioxide-capture material, carboxyl group modified 2D COF $\left(\left[\mathrm{HO}_{2} \mathrm{C}\right]_{x \%}-\mathrm{H}_{2} \mathrm{P}-\mathrm{COFs}\right)[64]$. The $\left[\mathrm{HO}_{2} \mathrm{C}\right]_{x \%}-\mathrm{H}_{2} \mathrm{P}-\mathrm{COFs}$ show micropores between 1.4-2.2 nm instead of the mesopores of $[\mathrm{HO}]_{x \%}-\mathrm{H}_{2} \mathrm{P}-\mathrm{COFs}(2.5 \mathrm{~nm})$. From the results of Table 3 , the $[\mathrm{HO}]_{x \%}-\mathrm{H}_{2} \mathrm{P}-\mathrm{COFs}$ have low $\mathrm{CO}_{2}$ capacity between $23-32 \mathrm{~cm}^{3} \cdot \mathrm{g}^{-1}$ at $273 \mathrm{~K}$ and 1 bar. However, $\left[\mathrm{HO}_{2} \mathrm{C}\right]_{x \%}-\mathrm{H}_{2} \mathrm{P}-\mathrm{COFs}$ exhibit a dramatically enhanced $\mathrm{CO}_{2}$ adsorption between $49-89 \mathrm{~m}^{3} \cdot \mathrm{g}^{-1}$ under the same conditions.

Jiang et al. also carried out a similar strategy to modify the pore surface with different functional groups through click reactions [66,67]. From Table 3, we can observe that different function of pore walls of COF materials can induce the decrease of surface area, pore sizes and pore volumes. However, the capacity for $\mathrm{CO}_{2}$ adsorption was highly dependent on the structures of the functional groups. Both from Table 3 and Figure 28, ethyl functionalized COFs, $[\mathrm{Et}]_{x}-\mathrm{H}_{2} \mathrm{P}-\mathrm{COFs}$, exhibit similar $\mathrm{CO}_{2}$ adsorption capacity to those of $\left[\mathrm{CH} \equiv \mathrm{C}_{\mathrm{x}}-\mathrm{H}_{2} \mathrm{P}-\mathrm{COFs}\right.$. The $\mathrm{CO}_{2}$ adsorption capacities of $[\mathrm{CH} \equiv \mathrm{C}]_{x}-\mathrm{H}_{2} \mathrm{P}-\mathrm{COFs}$ and $\left[\mathrm{EtNH}_{2}\right]_{x}-\mathrm{H}_{2} \mathrm{P}-\mathrm{COFs}$ enhanced with the increase of BET surface area and pore size. However, $[\mathrm{MeOAc}]_{x}-\mathrm{H}_{2} \mathrm{P}-\mathrm{COFs},[\mathrm{AcOH}]_{x}-\mathrm{H}_{2} \mathrm{P}-\mathrm{COFs},[\mathrm{EtOH}]_{x}-\mathrm{H}_{2} \mathrm{P}-\mathrm{COFs}$ and [EtNH $]_{x}-\mathrm{H}_{2} \mathrm{P}-\mathrm{COFs}$ show much higher $\mathrm{CO}_{2}$ adsorption capacity than those of $[\mathrm{CH} \equiv \mathrm{C}]_{x}-\mathrm{H}_{2} \mathrm{P}-\mathrm{COFs}$. The $\mathrm{CO}_{2}$ adsorption capacities are in order of $[\mathrm{CH} \equiv \mathrm{C}]_{x}-\mathrm{H}_{2} \mathrm{P}-\mathrm{COFs} \approx[\mathrm{Et}]_{x}-\mathrm{H}_{2} \mathrm{P}-\mathrm{COFs} \ll$ $[\mathrm{MeOAc}]_{x}-\mathrm{H}_{2} \mathrm{P}-\mathrm{COFs}<[\mathrm{AcOH}]_{x}-\mathrm{H}_{2} \mathrm{P}-\mathrm{COFs} \approx[\mathrm{EtOH}]_{x}-\mathrm{H}_{2} \mathrm{P}-\mathrm{COFs}<\left[\mathrm{EtNH}_{2}\right]_{x}-\mathrm{H}_{2} \mathrm{P}-\mathrm{COFs}$, which is due to the interaction between the functional group and $\mathrm{CO}_{2}$ molecule. These results indicate that the $\mathrm{CO}_{2}$ adsorption performances are highly related to the pore surface of the COF materials.
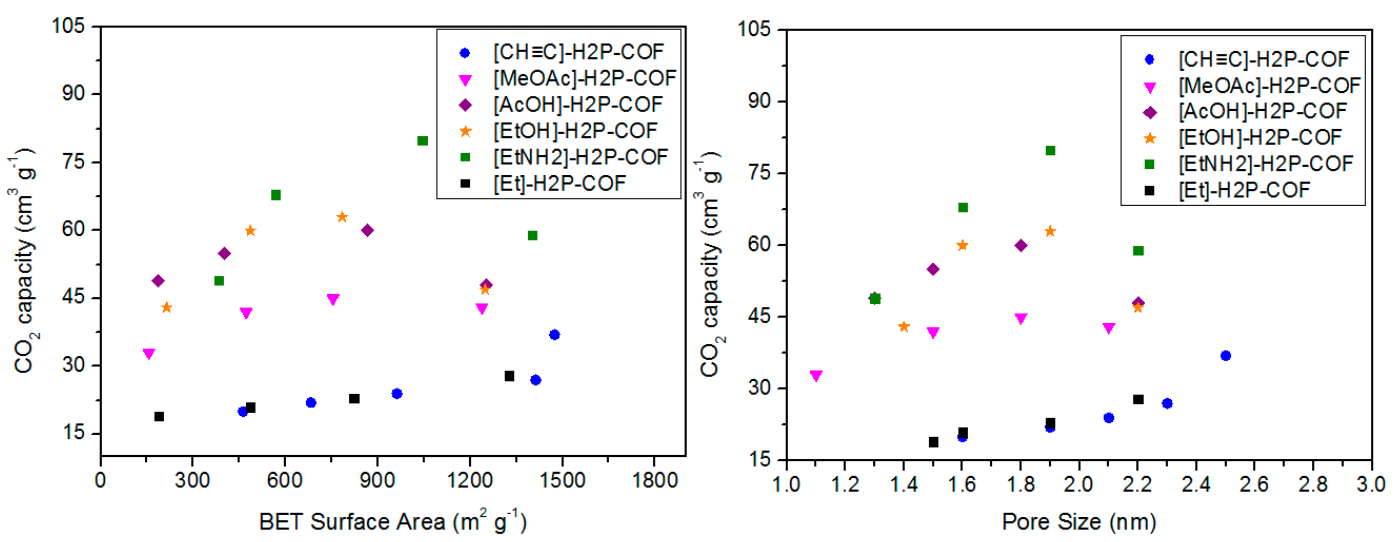

Figure 28. Plot of low-pressure $\mathrm{CO}_{2}$ uptake against BET surface area (left) and pore size (right) for the selected $\mathrm{COF}$ at $273 \mathrm{~K}$ and 1 bar.

\section{Perspectives and Challenges}

The past decade has been witnessed the blooming COF chemistry and their applications. Their unique features, such as flexible molecular structure design, permanent porosity, low density, modest to high crystallinity and controllable pore size from ultraporous to mesoporous, and the diversity of available building blocks, easily make them targets for structural and functional design, 
and promise their great potential as materials for application in various areas. However, there are still a lot of challenges in the design and synthesis of COFs.

First, compared to the high ordered structure of MOF materials, further investigation on the control of crystal growth, the skeletons, and pores is still of great importance. Currently, thermodynamically controlled solvothermal reactions are widely used to synthesize COF materials. The investigations reveal that the formation and growth of crystal nuclei to improve the crystallinity of COFs is quite important. Such studies provide a general pathway for the preparation of high crystallinity COFs. Moreover, control over the defect sites, the morphology and the stacking mode of COF's structures are also important and effective methods to construct precise crystal COF structures are necessary. At present, no direct analytical tools can be used to visualize and analyze the stacking and topological structures of COFs. Thus, the creation of single-crystal COFs an unattainable target.

Meanwhile, through the existing approaches, the methods to increase the surface area and porosities of COF materials are a big bottleneck. The surface area and porosities are the most important parameters for COF materials for gas adsorption and gas separation applications. How to design COFs structures which can improve both surface area and these properties is the key to realize the potential application of COFs in these fields. For the 2D COFs, spatially enlarging the interlayered spaces would increase the surface areas. For 3D COFs, the construction of highly crystalline frameworks would lead to very high BET surface areas that can reach the theoretical $S_{\mathrm{BET}}$ over $6000 \mathrm{~m}^{2} \cdot \mathrm{g}^{-1}$.

Acknowledgments: This work was supported by the National Natural Science Foundation of China (No. 21522105), 1000 Talent Plan (the Recruitment Program for Young Professionals), and ShanghaiTech University Research Startup Fund.

Author Contributions: L.Z. and Y.-B.Z. discussed and wrote this manuscript collaboratively. Y.-B.Z. led this work.

Conflicts of Interest: The authors declare no conflict of interest.

\section{References}

1. Waller, P.J.; Gandara, F.; Yaghi, O.M. Chemistry of covalent organic frameworks. Acc. Chem. Res. 2015, 48, 3053-3063. [CrossRef] [PubMed]

2. Ding, S.Y.; Wang, W. Covalent organic frameworks (COFs): From design to applications. Chem. Soc. Rev. 2013, 42, 548-568. [CrossRef] [PubMed]

3. Feng, X.; Ding, X.S.; Jiang, D.L. Covalent organic frameworks. Chem. Soc. Rev. 2012, 41, 6010-6022. [CrossRef] [PubMed]

4. Segura, J.L.; Mancheno, M.J.; Zamora, F. Covalent organic frameworks based on schiff-base chemistry: Synthesis, properties and potential applications. Chem. Soc. Rev. 2016, 45, 5635-5671. [CrossRef] [PubMed]

5. Diercks, C.S.; Yaghi, O.M. The atom, the molecule, and the covalent organic framework. Science 2017, 355, 6328. [CrossRef] [PubMed]

6. Rogge, S.M.J.; Bavykina, A.; Hajek, J.; Garcia, H.; Olivos-Suarez, A.I.; Sepulveda-Escribano, A.; Vimont, A.; Clet, G.; Bazin, P.; Kapteijn, F.; et al. Metal-organic and covalent organic frameworks as single-site catalysts. Chem. Soc. Rev. 2017, 46, 3134-3184. [CrossRef] [PubMed]

7. Rowan, S.J.; Cantrill, S.J.; Cousins, G.R.L.; Sanders, J.K.M.; Stoddart, J.F. Dynamic covalent chemistry. Angew. Chem. Int. Ed. 2002, 41, 898-952. [CrossRef]

8. Roy, N.; Lehn, J.M. Dynamic covalent chemistry: A facile room-temperature, reversible, diels-alder reaction between anthracene derivatives and n-phenyltriazolinedione. Chem.-Asian J. 2011, 6, 2419-2425. [CrossRef] [PubMed]

9. Beaudoin, D.; Maris, T.; Wuest, J.D. Constructing monocrystalline covalent organic networks by polymerization. Nat. Chem. 2013, 5, 830-834. [CrossRef] [PubMed]

10. Cote, A.P.; Benin, A.I.; Ockwig, N.W.; O'Keeffe, M.; Matzger, A.J.; Yaghi, O.M. Porous, crystalline, covalent organic frameworks. Science 2005, 310, 1166-1170. [CrossRef] [PubMed]

11. El-Kaderi, H.M.; Hunt, J.R.; Mendoza-Cortes, J.L.; Cote, A.P.; Taylor, R.E.; O’Keeffe, M.; Yaghi, O.M. Designed synthesis of $3 d$ covalent organic frameworks. Science 2007, 316, 268-272. [CrossRef] [PubMed] 
12. Campbell, N.L.; Clowes, R.; Ritchie, L.K.; Cooper, A.I. Rapid microwave synthesis and purification of porous covalent organic frameworks. Chem. Mater. 2009, 21, 204-206. [CrossRef]

13. Cote, A.P.; El-Kaderi, H.M.; Furukawa, H.; Hunt, J.R.; Yaghi, O.M. Reticular synthesis of microporous and mesoporous 2d covalent organic frameworks. J. Am. Chem. Soc. 2007, 129, 12914-12915. [CrossRef] [PubMed]

14. Wan, S.; Guo, J.; Kim, J.; Ihee, H.; Jiang, D.L. A photoconductive covalent organic framework: Self-condensed arene cubes composed of eclipsed 2d polypyrene sheets for photocurrent generation. Angew. Chem. Int. Ed. 2009, 48, 5439-5442. [CrossRef] [PubMed]

15. Tilford, R.W.; Mugavero, S.J.; Pellechia, P.J.; Lavigne, J.J. Tailoring microporosity in covalent organic frameworks. Adv. Mater. 2008, 20, 2741-2746. [CrossRef] [PubMed]

16. Lanni, L.M.; Tilford, R.W.; Bharathy, M.; Lavigne, J.J. Enhanced hydrolytic stability of self-assembling alkylated two-dimensional covalent organic frameworks. J. Am. Chem. Soc. 2011, 133, 13975-13983. [CrossRef] [PubMed]

17. Dogru, M.; Sonnauer, A.; Gavryushin, A.; Knochel, P.; Bein, T. A covalent organic framework with $4 \mathrm{~nm}$ open pores. Chem. Commun. 2011, 47, 1707-1709. [CrossRef] [PubMed]

18. Spitler, E.L.; Colson, J.W.; Uribe-Romo, F.J.; Woll, A.R.; Giovino, M.R.; Saldivar, A.; Dichtel, W.R. Lattice expansion of highly oriented $2 \mathrm{~d}$ phthalocyanine covalent organic framework films. Angew. Chem. Int. Ed. 2012, 51, 2623-2627. [CrossRef] [PubMed]

19. Colson, J.W.; Mann, J.A.; DeBlase, C.R.; Dichtel, W.R. Patterned growth of oriented 2d covalent organic framework thin films on single-layer graphene. J. Polym. Sci. Polym. Chem. 2015, 53, 378-384. [CrossRef]

20. Spitler, E.L.; Dichtel, W.R. Lewis acid-catalysed formation of two-dimensional phthalocyanine covalent organic frameworks. Nat. Chem. 2010, 2, 672-677. [CrossRef] [PubMed]

21. Spitler, E.L.; Giovino, M.R.; White, S.L.; Dichtel, W.R. A mechanistic study of lewis acid-catalyzed covalent organic framework formation. Chem. Sci. 2011, 2, 1588-1593. [CrossRef]

22. Dogru, M.; Sonnauer, A.; Zimdars, S.; Doblinger, M.; Knochel, P.; Bein, T. Facile synthesis of a mesoporous benzothiadiazole-cof based on a transesterification process. Crystengcomm 2013, 15, 1500-1502. [CrossRef]

23. Calik, M.; Sick, T.; Dogru, M.; Doblinger, M.; Datz, S.; Budde, H.; Hartschuh, A.; Auras, F.; Bein, T. From highly crystalline to outer surface-functionalized covalent organic frameworks-a modulation approach. J. Am. Chem. Soc. 2016, 138, 1234-1239. [CrossRef] [PubMed]

24. Du, Y.; Yang, H.S.; Whiteley, J.M.; Wan, S.; Jin, Y.H.; Lee, S.H.; Zhang, W. Ionic covalent organic frameworks with spiroborate linkage. Angew. Chem. Int. Ed. 2016, 55, 1737-1741. [CrossRef] [PubMed]

25. Uribe-Romo, F.J.; Hunt, J.R.; Furukawa, H.; Klock, C.; O’Keeffe, M.; Yaghi, O.M. A crystalline imine-linked 3-d porous covalent organic framework. J. Am. Chem. Soc. 2009, 131, 4570-4571. [CrossRef] [PubMed]

26. Zhang, Y.B.; Su, J.; Furukawa, H.; Yun, Y.F.; Gandara, F.; Duong, A.; Zou, X.D.; Yaghi, O.M. Single-crystal structure of a covalent organic framework. J. Am. Chem. Soc. 2013, 135, 16336-16339. [CrossRef] [PubMed]

27. Ma, Y.X.; Li, Z.J.; Wei, L.; Ding, S.Y.; Zhang, Y.B.; Wang, W. A dynamic three-dimensional covalent organic framework. J. Am. Chem. Soc. 2017, 139, 4995-4998. [CrossRef] [PubMed]

28. Lin, G.Q.; Ding, H.M.; Yuan, D.Q.; Wang, B.S.; Wang, C. A pyrene-based, fluorescent three-dimensional covalent organic framework. J. Am. Chem. Soc. 2016, 138, 3302-3305. [CrossRef] [PubMed]

29. Fang, Q.R.; Gu, S.; Zheng, J.; Zhuang, Z.B.; Qiu, S.L.; Yan, Y.S. 3D microporous base-functionalized covalent organic frameworks for size-selective catalysis. Angew. Chem. Int. Ed. 2014, 53, 2878-2882. [CrossRef] [PubMed]

30. Kandambeth, S.; Shinde, D.B.; Panda, M.K.; Lukose, B.; Heine, T.; Banerjee, R. Enhancement of chemical stability and crystallinity in porphyrin-containing covalent organic frameworks by intramolecular hydrogen bonds. Angew. Chem. Int. Ed. 2013, 52, 13052-13056. [CrossRef] [PubMed]

31. Chen, X.; Addicoat, M.; Jin, E.; Zhai, L.; Xu, H.; Huang, N.; Guo, Z.; Liu, L.; Irle, S.; Jiang, D. Locking covalent organic frameworks with hydrogen bonds: General and remarkable effects on crystalline structure, physical properties, and photochemical activity. J. Am. Chem. Soc. 2015, 137, 3241-3247. [CrossRef] [PubMed]

32. Chen, X.; Addicoat, M.; Irle, S.; Nagai, A.; Jiang, D. Control of crystallinity and porosity of covalent organic frameworks by managing interlayer interactions based on self-complementary $\pi$-electronic force. J. Am. Chem. Soc. 2013, 135, 546-549. [CrossRef] [PubMed]

33. Xu, H.; Tao, S.S.; Jiang, D.L. Proton conduction in crystalline and porous covalent organic frameworks. Nat. Mater. 2016, 15, 722-726. [CrossRef] [PubMed] 
34. Ascherl, L.; Sick, T.; Margraf, J.T.; Lapidus, S.H.; Calik, M.; Hettstedt, C.; Karaghiosoff, K.; Doblinger, M.; Clark, T.; Chapman, K.W.; et al. Molecular docking sites designed for the generation of highly crystalline covalent organic frameworks. Nat. Chem. 2016, 8, 310-316. [CrossRef]

35. Auras, F.; Ascherl, L.; Haldmioun, A.H.; Margraf, J.T.; Hanusch, F.C.; Reuter, S.; Bessinger, D.; Doblinger, M.; Hettstedt, C.; Karaghiosoff, K.; et al. Synchronized offset stacking: A concept for growing large-domain and highly crystalline $2 \mathrm{~d}$ covalent organic frameworks. J. Am. Chem. Soc. 2016, 138, 16703-16710. [CrossRef] [PubMed]

36. Kandambeth, S.; Mallick, A.; Lukose, B.; Mane, M.V.; Heine, T.; Banerjee, R. Construction of crystalline 2d covalent organic frameworks with remarkable chemical (acid/base) stability via a combined reversible and irreversible route. J. Am. Chem. Soc. 2012, 134, 19524-19527. [CrossRef] [PubMed]

37. Biswal, B.P.; Chandra, S.; Kandambeth, S.; Lukose, B.; Heine, T.; Banerjeet, R. Mechanochemical synthesis of chemically stable isoreticular covalent organic frameworks. J. Am. Chem. Soc. 2013, 135, 5328-5331. [CrossRef] [PubMed]

38. Waller, P.J.; Lyle, S.J.; Popp, T.M.O.; Diercks, C.S.; Reimer, J.A.; Yaghi, O.M. Chemical conversion of linkages in covalent organic frameworks. J. Am. Chem. Soc. 2016, 138, 15519-15522. [CrossRef] [PubMed]

39. Uribe-Romo, F.J.; Doonan, C.J.; Furukawa, H.; Oisaki, K.; Yaghi, O.M. Crystalline covalent organic frameworks with hydrazone linkages. J. Am. Chem. Soc. 2011, 133, 11478-11481. [CrossRef] [PubMed]

40. Bunck, D.N.; Dichtel, W.R. Bulk synthesis of exfoliated two-dimensional polymers using hydrazone-linked covalent organic frameworks. J. Am. Chem. Soc. 2013, 135, 14952-14955. [CrossRef] [PubMed]

41. Stegbauer, L.; Schwinghammer, K.; Lotsch, B.V. A hydrazone-based covalent organic framework for photocatalytic hydrogen production. Chem. Sci. 2014, 5, 2789-2793. [CrossRef]

42. Ding, S.Y.; Dong, M.; Wang, Y.W.; Chen, Y.T.; Wang, H.Z.; Su, C.Y.; Wang, W. Thioether-based fluorescent covalent organic framework for selective detection and facile removal of mercury(ii). J. Am. Chem. Soc. 2016, 138, 3031-3037. [CrossRef] [PubMed]

43. Dalapati, S.; Jin, S.B.; Gao, J.; Xu, Y.H.; Nagai, A.; Jiang, D.L. An azine-linked covalent organic framework. J. Am. Chem. Soc. 2013, 135, 17310-17313. [CrossRef] [PubMed]

44. Li, Z.P.; Feng, X.; Zou, Y.C.; Zhang, Y.W.; Xia, H.; Liu, X.M.; Mu, Y. A 2d azine-linked covalent organic framework for gas storage applications. Chem. Commun. 2014, 50, 13825-13828. [CrossRef] [PubMed]

45. Li, Z.P.; Zhi, Y.F.; Feng, X.; Ding, X.S.; Zou, Y.C.; Liu, X.M.; Mu, Y. An azine-linked covalent organic framework: Synthesis, characterization and efficient gas storage. Chem.-Eur. J. 2015, 21, 12079-12084. [CrossRef] [PubMed]

46. Alahakoon, S.B.; Thompson, C.M.; Nguyen, A.X.; Occhialini, G.; McCandless, G.T.; Smaldone, R.A. An azine-linked hexaphenylbenzene based covalent organic framework. Chem. Commun. 2016, 52, 2843-2845. [CrossRef] [PubMed]

47. Vyas, V.S.; Haase, F.; Stegbauer, L.; Savasci, G.; Podjaski, F.; Ochsenfeld, C.; Lotsch, B.V. A tunable azine covalent organic framework platform for visible light-induced hydrogen generation. Nat. Commun. 2015, 6, 8508-8516. [CrossRef] [PubMed]

48. Zhang, J.; Wang, L.B.; Li, N.; Liu, J.F.; Zhang, W.; Zhang, Z.B.; Zhou, N.C.; Zhu, X.L. A novel azobenzene covalent organic framework. Crystengcomm 2014, 16, 6547-6551. [CrossRef]

49. Nagai, A.; Chen, X.; Feng, X.; Ding, X.S.; Guo, Z.Q.; Jiang, D.L. A squaraine-linked mesoporous covalent organic framework. Angew. Chem. Int. Ed. 2013, 52, 3770-3774. [CrossRef] [PubMed]

50. Fang, Q.R.; Wang, J.H.; Gu, S.; Kaspar, R.B.; Zhuang, Z.B.; Zheng, J.; Guo, H.X.; Qiu, S.L.; Yan, Y.S. 3d porous crystalline polyimide covalent organic frameworks for drug delivery. J. Am. Chem. Soc. 2015, 137, 8352-8355. [CrossRef] [PubMed]

51. Fang, Q.R.; Zhuang, Z.B.; Gu, S.; Kaspar, R.B.; Zheng, J.; Wang, J.H.; Qiu, S.L.; Yan, Y.S. Designed synthesis of large-pore crystalline polyimide covalent organic frameworks. Nat. Commun. 2014, 5, 4503-4510. [CrossRef] [PubMed]

52. Kou, Y.; Xu, Y.H.; Guo, Z.Q.; Jiang, D.L. Supercapacitive energy storage and electric power supply using an aza-fused pi-conjugated microporous framework. Angew. Chem. Int. Edit. 2011, 50, 8753-8757. [CrossRef] [PubMed]

53. Guo, J.; Xu, Y.H.; Jin, S.B.; Chen, L.; Kaji, T.; Honsho, Y.; Addicoat, M.A.; Kim, J.; Saeki, A.; Ihee, H.; et al. Conjugated organic framework with three-dimensionally ordered stable structure and delocalized pi clouds. Nat. Commun. 2013, 4, 2736-2743. [CrossRef] [PubMed] 
54. Kuhn, P.; Antonietti, M.; Thomas, A. Porous, covalent triazine-based frameworks prepared by ionothermal synthesis. Angew. Chem. Int. Ed. 2008, 47, 3450-3453. [CrossRef] [PubMed]

55. Kuhn, P.; Forget, A.; Su, D.; Thomas, A.; Antonietti, M. From microporous regular frameworks to mesoporous materials with ultrahigh surface area: Dynamic reorganization of porous polymer networks. J. Am. Chem. Soc. 2008, 130, 13333-13337. [CrossRef] [PubMed]

56. Bojdys, M.J.; Jeromenok, J.; Thomas, A.; Antonietti, M. Rational extension of the family of layered, covalent, triazine-based frameworks with regular porosity. Adv. Mater. 2010, 22, 2202-2205. [CrossRef] [PubMed]

57. Ren, S.J.; Bojdys, M.J.; Dawson, R.; Laybourn, A.; Khimyak, Y.Z.; Adams, D.J.; Cooper, A.I. Porous, fluorescent, covalent triazine-based frameworks via room-temperature and microwave-assisted synthesis. Adv. Mater. 2012, 24, 2357-2361. [CrossRef] [PubMed]

58. Katekomol, P.; Roeser, J.; Bojdys, M.; Weber, J.; Thomas, A. Covalent triazine frameworks prepared from 1,3,5-tricyanobenzene. Chem. Mater. 2013, 25, 1542-1548. [CrossRef]

59. Karmakar, A.; Kumar, A.; Chaudhari, A.K.; Samanta, P.; Desai, A.V.; Krishna, R.; Ghosh, S.K. Bimodal functionality in a porous covalent triazine framework by rational integration of an electron-rich and -deficient pore surface. Chem.-Eur. J. 2016, 22, 4931-4937. [CrossRef] [PubMed]

60. Zeng, Y.F.; Zou, R.Y.; Luo, Z.; Zhang, H.C.; Yao, X.; Ma, X.; Zou, R.Q.; Zhao, Y.L. Covalent organic frameworks formed with two types of covalent bonds based on orthogonal reactions. J. Am. Chem. Soc. 2015, 137, 1020-1023. [CrossRef] [PubMed]

61. Li, H.; Pan, Q.Y.; Ma, Y.C.; Guan, X.Y.; Xue, M.; Fang, Q.R.; Yan, Y.S.; Valtchev, V.; Qiu, S.L. Three-dimensional covalent organic frameworks with dual linkages for bifunctional cascade catalysis. J. Am. Chem. Soc. 2016, 138, 14783-14788. [CrossRef] [PubMed]

62. Furukawa, H.; Yaghi, O.M. Storage of hydrogen, methane, and carbon dioxide in highly porous covalent organic frameworks for clean energy applications. J. Am. Chem. Soc. 2009, 131, 8875-8883. [CrossRef] [PubMed]

63. Cao, D.; Lan, J.; Wang, W.; Smit, B. Lithium-doped 3D covalent organic frameworks: High-capacity hydrogen storage materials. Angew. Chem. Int. Ed. 2009, 48, 4730-4733. [CrossRef] [PubMed]

64. Klontzas, E.; Tylianakis, E.; Froudakis, G.E. Hydrogen storage in lithium-functionalized 3-D covalent-organic framework materials. J. Phys. Chem. C 2009, 113, 21253-21257. [CrossRef]

65. Zhao, Y.; Yao, K.X.; Teng, B.; Zhang, T.; Han, Y. A perfluorinated covalent triazine-based framework for highly selective and water-tolerant $\mathrm{CO}_{2}$ capture. Energy Environ. Sci. 2013, 6, 3684-3692. [CrossRef]

66. Huang, N.; Chen, X.; Krishna, R.; Jiang, D. Two-dimensional covalent organic frameworks for carbon dioxide capture through channel-wall functionalization. Angew. Chem. Int. Ed. 2015, 54, 2986-2990. [CrossRef] [PubMed]

67. Huang, N.; Krishna, R.; Jiang, D. Tailor-made pore surface engineering in covalent organic frameworks: Systematic functionalization for performance screening. J. Am. Chem. Soc. 2015, 137, 7079-7082. [CrossRef] [PubMed]

(C) 2017 by the authors. Licensee MDPI, Basel, Switzerland. This article is an open access article distributed under the terms and conditions of the Creative Commons Attribution (CC BY) license (http:// creativecommons.org/licenses/by/4.0/). 